Supporting Information

\title{
Stereochemical Determination of Archazolid A and B, Highly Potent V-ATPase Inhibitors from the Myxobacterium Archangium gephyra
}

Jorma Hassfeld, ${ }^{\dagger}$ Christophe Farès,${ }^{\S}$ Heinrich Steinmetz, ${ }^{\ddagger}$ Teresa Carlomagno, ${ }^{\S}$ and Dirk Menche*,†

${ }^{\dagger}$ Gesellschaft für Biotechnologische Forschung mbH, Medzinische Chemie, Mascheroder Weg 1, D-38124 Braunschweig, Germany, ${ }^{\ddagger}$ Gesellschaft für Biotechnologische Forschung $\mathrm{mbH}$, Umweltmikrobiologie, Mascheroder Weg 1, D-38124 Braunschweig, Germany and ${ }^{\ddagger}$ Max Planck Institute for Biophysical Chemistry, Department of NMR based Structural Biology, Am Fassberg, 11, D-37077 Göttingen.

dme05@gbf.de

\section{Contents}

I. Tables of NMR Data and experimental procedures

II. Copies of 1D and 2D NMR spectra

III. Molecular Modeling

\section{Tables of NMR Data and experimental procedures}

\section{General experimental}

Nuclear magnetic resonance (NMR) spectra were recorded on a Bruker AM 600 spectrometer and on a Bruker AVANCE 900 spectrometer operating at a proton frequency of 600.13 and 900.00 MHz. Proton spectra were referenced to 3.34 for residual $\mathrm{CHD}_{2} \mathrm{OD}$. The ROESY experiments were acquired with mixing times of $500 \mathrm{~ms}$. Acquisition parameters included $1 \mathrm{~K}$ data points in $F_{1}$ for processing. The NOESY experiments were acquired with mixing times of 50-700ms. Acquisition parameters included $2 \mathrm{~K}$ data points in $\mathrm{F}_{2}$ and 512 increments in $\mathrm{F}_{1}$. The data was zero-filled to $4 \mathrm{~K}$ in $\mathrm{F}_{2}$ and $1 \mathrm{~K}$ in $\mathrm{F}_{1}$. The HSQC-HECADE experiment was acquired with a TOCSY mixing time of $60-80 \mathrm{msec}$ and a $J$ (scale) factor of 2 . The acquisition parameters included $4 \mathrm{~K}$ data points in $\mathrm{F}_{2}$ and 256 increments with 64 scans per increment. The data was zero-filled to $8 \mathrm{~K}_{\text {in }} \mathrm{F}_{2}$ and $1 \mathrm{~K}_{\text {in }} \mathrm{F}_{1}$. 
Table 1. ${ }^{1} \mathrm{H}$-NMR data and ROESY Correlations for archazolid A (600 $\left.\mathrm{MHz}, \mathrm{CD}_{3} \mathrm{OD}\right)$

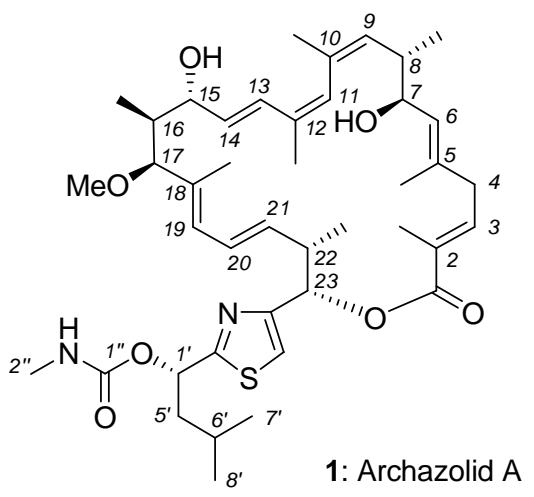

\begin{tabular}{|c|c|c|}
\hline $\mathbf{H}$ & $\delta$ [ppm], mult., $J[\mathrm{~Hz}]$ & ROESY Correlations $^{\mathrm{a}}$ \\
\hline 3 & 6.82, ddd, $7.9 / 7.5 / 1.3$ & $\mathrm{H}-4 \mathrm{a}(\mathrm{s}), \mathrm{H}-4 \mathrm{~b}(\mathrm{~s}), 6-\mathrm{H}(\mathrm{m}), \mathrm{Me}-5$ (s), Me-2 (m), H-21 (w) \\
\hline $4 \mathrm{a}$ & $2.91, \mathrm{dd}, 15.0 / 7.5$ & H-4b (vs), H-3 (s), 6-H (s), Me-2 (s), Me-5 (m) \\
\hline $4 \mathrm{~b}$ & $3.03, \mathrm{dd}, 15.0 / 7.5$ & $\mathrm{H}-4 \mathrm{a}(\mathrm{vs}), \mathrm{H}-3(\mathrm{~s}), 6-\mathrm{H}(\mathrm{s}), \mathrm{Me}-2$ (s), Me-5 (m) \\
\hline 6 & $5.21, \mathrm{dd}, 9.5 / 1.2$ & $\begin{array}{l}\text { H-4a (s), H-4b (s), H-8 (s), H-3 (m), H-7 (m), Me-3 (m), } \\
\text { Me-5 (m), Me-8 (m), Me-16 (m) }\end{array}$ \\
\hline 7 & $4.03, \mathrm{dd}, 9.4 / 9.3$ & Me-5 (s), H-9 (s), Me-8 (s), H-6 (m), H-8 (m) \\
\hline 8 & 2.30, ddq, $9.5 / 9.8 / 7.0$ & H-6 (s), H-7 (m), H-9 (m), H-11 (m), H-13 (m), Me-8 (s) \\
\hline 9 & $5.27, \mathrm{~d}, 9.6$ & $\mathrm{H}-7$ (s), Me-8 (s), Me-10 (s), H-8 (m) \\
\hline 11 & $5.81, \mathrm{~d}, 1.0$ & $\operatorname{Me}-12(\mathrm{~s}), \mathrm{H}-8(\mathrm{~m}), \mathrm{Me}-8(\mathrm{w}), \mathrm{Me}-10(\mathrm{~m})$ \\
\hline 13 & $6.56, \mathrm{dd}, 15.5 / 0.9$ & $\begin{array}{l}\text { H-15 (s), H-8 (m), H-14 (m), Me-8 (m), Me-10 (m), H-16 } \\
(w), M e-16(w)\end{array}$ \\
\hline 14 & $5.79, \mathrm{dd}, 15.5 / 6.0$ & $\mathrm{H}-13(\mathrm{~m}), \mathrm{H}-15(\mathrm{~s}), \mathrm{H}-16(\mathrm{~s}), \mathrm{Me}-12(\mathrm{~s}), \mathrm{Me}-16(\mathrm{~m})$ \\
\hline 15 & $4.31, \mathrm{dd}, 6.4 / 3.2$ & $\mathrm{H}-13(\mathrm{~s}), \mathrm{H}-14(\mathrm{~s}), \mathrm{H}-16(\mathrm{~s}), \mathrm{Me}-16(\mathrm{~m})$ \\
\hline 16 & 1.80, ddq, $9.0 / 3.2 / 7.0$ & $\mathrm{H}-14(\mathrm{~s}), \mathrm{H}-15(\mathrm{~s}), \mathrm{H}-13(\mathrm{w})$ \\
\hline 17 & $3.40, \mathrm{~d}, 9.0$ & $\mathrm{H}-19(\mathrm{~s}), \mathrm{Me}-16(\mathrm{~m}), \mathrm{Me}-18(\mathrm{~m})$ \\
\hline 19 & $5.87, \mathrm{dd}, 10.9 / 1.2$ & $\begin{array}{l}\text { H-17 (s), H-21 (s), H-20 (m), Me-8 (m), Me-16 (m), Me-18 } \\
(\mathrm{m}), \text { OMe-17 (m) }\end{array}$ \\
\hline 20 & $6.17, \mathrm{dd}, 15.5 / 10.9$ & H-22 (s), Me-18 (s), Me- 22 (s), H-19 (m), H-21 (m), \\
\hline 21 & $5.63, \mathrm{dd}, 15.2 / 7.0$ & H-19 (s), H-22 (s), H-20 (m), Me-22 (m), H-3 (w), H-4' (w) \\
\hline 22 & $3.10, \mathrm{ddq}, 7.5 / 4.0 / 7.0$ & $\mathrm{H}-20(\mathrm{~s}), \mathrm{H}-21(\mathrm{~s}), \mathrm{H}-23(\mathrm{~s}), \mathrm{Me}-8(\mathrm{~m}), \mathrm{H}-4 \mathrm{f}^{\prime}(\mathrm{w})$ \\
\hline 23 & $5.97, \mathrm{~d}, 4.1$ & $\mathrm{H}-22(\mathrm{~s}), \mathrm{Me}-22(\mathrm{~s}), \mathrm{H}-4^{\prime}(\mathrm{m})$ \\
\hline $\mathrm{Me}-2$ & $1.91, \mathrm{~d}, 1.2$ & $\mathrm{H}-4 \mathrm{a}(\mathrm{s}), \mathrm{H}-4 \mathrm{~b}(\mathrm{~s}), \mathrm{H}-3(\mathrm{~m})$ \\
\hline $\mathrm{Me}-5$ & $1.73, \mathrm{~d}, 1.2$ & H-7 (s), H-3 (m), H-4a (m), H-4b (m), H-6 (w) \\
\hline Me-8 & $0.84, \mathrm{~d}, 7.0$ & $\begin{array}{l}\text { H-7 (s), H-8 (s), H-9 (s), H-6 (m), H-13 (m), H-19 (m), H-22 } \\
(\mathrm{m}), \mathrm{H}-11(\mathrm{w}),\end{array}$ \\
\hline Me-10 & 1.80, brs & $\mathrm{H}-9(\mathrm{~s}), \mathrm{H}-11(\mathrm{~m}), \mathrm{H}-13(\mathrm{~m})$ \\
\hline $\mathrm{Me}-12$ & $1.93, \mathrm{~d}, 1.2$ & $\mathrm{H}-11(\mathrm{~s}), \mathrm{H}-14(\mathrm{~s})$ \\
\hline Me-16 & $0.74, \mathrm{~d}, 7.0$ & $\begin{array}{l}\text { Me-18 (s), H-6 (m), H-14 (m), H-15 (m), H-17 (m), H-19 } \\
(\mathrm{m}), \mathrm{H}-13 \text { (w) }\end{array}$ \\
\hline OMe-17 & $3.16, \mathrm{~s}$ & $\mathrm{H}-19(\mathrm{~m})$ \\
\hline Me-18 & $1.64, \mathrm{~d}, 1.2$ & H-20 (s), Me-16 (s), H-17 (m), H-19 (m) \\
\hline Me-22 & $1.15, \mathrm{~d}, 7.0$ & $\mathrm{H}-20(\mathrm{~s}), \mathrm{H}-22(\mathrm{~s}), \mathrm{H}-23(\mathrm{~s}), \mathrm{H}-21(\mathrm{~m}), \mathrm{H}-4^{\prime}(\mathrm{w})$ \\
\hline $1^{\prime}$ & $6.04, \mathrm{dd}, 9.1 / 4.5$ & H-5' (m), Me-7' (w), Me-8' (w) \\
\hline $4^{\prime}$ & $7.21, \mathrm{~s}$ & $\mathrm{H}-23(\mathrm{~m}), \mathrm{H}-21$ (w), H-22 (w), Me-22 (w) \\
\hline $5^{\prime}$ & $1.92, \mathrm{~m}$ & H-1' (m), H-6' (m) \\
\hline $6^{\prime}$ & $1.77, \mathrm{~m}$ & Me-7' (s), Me-8' (s), H-5' (m) \\
\hline Me-7' & $1.01, \mathrm{~d}, 6.0$ & H-6' (s), H-5' (m) \\
\hline Me-8' & $1.02, \mathrm{~d}, 6.0$ & H-6' (s), H-5' (m) \\
\hline $2 "$ & $2.75, \mathrm{~s}$ & $\mathrm{Me}-7^{\prime}(\mathrm{w}), \mathrm{Me}-8^{\prime}(\mathrm{w})$ \\
\hline
\end{tabular}

adefinitions: (s) = strong, $(\mathrm{m})=$ medium, $(\mathrm{s})=$ weak 
Table 2. Key ${ }^{1} \mathrm{H}$-NMR and ${ }^{1} \mathrm{C}$-NMR chemical shifts for archazolid $\mathrm{A}$ in $\mathrm{CD}_{3} \mathrm{OD}(600$ MHz) and DMSO-d 6 (900 MHz,

\begin{tabular}{|c|c|c|c|c|c|}
\hline $\mathbf{H}$ & $\begin{array}{c}\delta[\mathrm{ppm}] \text { in } \\
\mathrm{CD}_{3} \mathrm{OD}\end{array}$ & $\begin{array}{l}\delta \text { [ppm] in } \\
\text { DMSO-d }_{6}\end{array}$ & $\mathbf{C}$ & $\begin{array}{c}\delta\left[\mathrm{ppm}^{\mathrm{p}} \text { in }\right. \\
\mathrm{CD}_{3} \mathrm{OD}\end{array}$ & $\begin{array}{l}\delta[p p m] \text { in } \\
\text { DMSO-d }\end{array}$ \\
\hline 3 & 6.82 & 6.62 & 3 & 142.1 & 140.50 \\
\hline $4 a$ & 2.9 & 2.78 & 4 & 40.6 & 39.38 \\
\hline $4 \mathrm{~b}$ & 3.03 & 2.92 & 6 & 130.4 & 130.30 \\
\hline 6 & 5.21 & 5.06 & 7 & 73.6 & 71.49 \\
\hline 7 & 4.03 & 3.86 & 8 & 41.6 & 40.28 \\
\hline $7-\mathrm{OH}$ & & 4.53 & 9 & 132.7 & 132.70 \\
\hline 8 & 2.30 & 2.14 & 11 & 130.8 & 129.20 \\
\hline 9 & 5.27 & 5.20 & 13 & 129.3 & 127.00 \\
\hline 11 & 5.81 & 5.65 & 14 & 133.6 & 134.30 \\
\hline 13 & 6.56 & 6.44 & 15 & 75.5 & 72.36 \\
\hline 14 & 5.79 & 5.69 & 16 & 44.5 & 43.16 \\
\hline 15 & 4.31 & 4.19 & 17 & 89.8 & 87.51 \\
\hline $15-\mathrm{OH}$ & & 4.42 & 19 & 129.9 & 128.00 \\
\hline 16 & 1.80 & 1.57 & 20 & 127.6 & 126.30 \\
\hline 17 & 3.40 & 3.29 & 21 & 135.1 & 133.70 \\
\hline 19 & 5.87 & 5.77 & 22 & 42.0 & 39.95 \\
\hline 20 & 6.17 & 6.13 & 23 & 77.6 & 75.96 \\
\hline 21 & 5.63 & 5.57 & $\mathrm{Me}-2$ & 12.6 & 12.57 \\
\hline 22 & 3.10 & 3.02 & $\mathrm{Me}-5$ & 16.8 & 16.60 \\
\hline 23 & 5.97 & 5.82 & $\mathrm{Me}-8$ & 17.7 & 17.24 \\
\hline $\mathrm{Me}-2$ & 1.91 & 1.81 & Me-10 & 24.7 & 24.83 \\
\hline $\mathrm{Me}-5$ & 1.73 & 1.59 & Me-12 & 19.9 & 19.81 \\
\hline Me-8 & 0.84 & 0.70 & Me-16 & 12.6 & 11.49 \\
\hline Me-10 & 1.80 & 1.70 & OMe-17 & 56.2 & 55.63 \\
\hline $\mathrm{Me}-12$ & 1.93 & 1.81 & Me-18 & 13.0 & 12.86 \\
\hline Me-16 & 0.74 & 0.56 & $\mathrm{Me}-22$ & 17.6 & 17.05 \\
\hline OMe-17 & 3.16 & 3.01 & $1^{\prime}$ & 73.3 & 70.99 \\
\hline Me-18 & 1.64 & 1.55 & $4^{\prime}$ & 116.7 & 116.40 \\
\hline Me-22 & 1.15 & 1.06 & $5^{\prime}$ & 46.0 & 44.32 \\
\hline $1^{\prime}$ & 6.04 & 5.87 & $6^{\prime}$ & 25.8 & 24.42 \\
\hline $4^{\prime}$ & 7.21 & 7.30 & $7^{\prime}$ & 23.4 & 23.02 \\
\hline 5'a & 1.92 & 1.76 & $8^{\prime}$ & 22.4 & 22.07 \\
\hline $5^{\prime} \mathrm{b}$ & 1.92 & 1.81 & $1 "$ & 158.2 & 155.91 \\
\hline $6^{\prime}$ & 1.77 & 1.70 & $2 "$ & 27.5 & 27.27 \\
\hline
\end{tabular}

Table 3. Relevant Homo- and Heteronuclear Coupling Constants for archazolid A (900 MHz, DMSO-d $\mathbf{d}_{6}$ )

\begin{tabular}{|l|l|l|l|}
\hline $\mathbf{H}$ & $\boldsymbol{\delta}[\mathbf{p p m}]$, mult., $\boldsymbol{J}[\mathbf{H z}]$ & ${ }^{2} \boldsymbol{J}_{\mathbf{H}, \mathbf{C}}[\mathbf{H z}]^{\mathbf{a}}$ & ${ }^{3} \boldsymbol{J}_{\mathbf{H}, \mathbf{C}}[\mathbf{H z}]^{\mathbf{a}}$ \\
\hline 6 & $5.21, \mathrm{dd}, 9.5 / 1.2$ & & $\mathrm{C}-8(2.6)$ \\
\hline 7 & $4.03, \mathrm{dd}, 9.4 / 9.3$ & $\mathrm{C}-8(-2.5)$ & $\mathrm{C}-9(1.7), \mathrm{Me}-8(1.9)$ \\
\hline 8 & $2.30, \mathrm{ddq}, 9.5 / 7.0 / 9.8$ & $\mathrm{C}-7(-5.5)$ & $\mathrm{C}-6(3.1)$ \\
\hline 9 & $5.27, \mathrm{~d}, 9.6$ & & $\mathrm{C}-7(2.2), \mathrm{Me}-8(2.1)$ \\
\hline 15 & $4.31, \mathrm{dd}, 6.4 / 3.2$ & $\mathrm{C}-16(-5.0)$ & $\mathrm{C}-17(2.2), \mathrm{Me}-16(4.3)$ \\
\hline 16 & $1.80, \mathrm{ddq}, 9.0 / 3.2 / 7.0$ & $\mathrm{C}-15(-2.1), \mathrm{C}-17(-7.2)$ & $\mathrm{C}-14(2.3)$ \\
\hline 17 & $3.40, \mathrm{~d}, 9.0$ & $\mathrm{C}-16(-3.4)$ & $\mathrm{Me}-16(1.1)$ \\
\hline 21 & $5.63, \mathrm{dd}, 15.2 / 7.0$ & $\mathrm{Me}-22(-3.6)$ & $\mathrm{C}-23(1.8)$ \\
\hline 22 & $3.10, \mathrm{ddq}, 7.5 / 4.0 / 7.0$ & $\mathrm{C}-23(-3.2)$ & $\mathrm{C}-18(\sim 0.2), \mathrm{C}-3$ ' (3.7) \\
\hline 23 & $5.97, \mathrm{~d}, 4.1$ & $\mathrm{C}-22(-3.6)$ & $\mathrm{C}-21(5.0), \mathrm{Me}-22(2.9)$ \\
\hline
\end{tabular}

${ }^{\mathrm{a}}$ Couplings determined by HSQC-HECADE at $900 \mathrm{MHz}$ 
Table 4. ${ }^{1} \mathrm{H}$-NMR data for the bis-Mosher esters of archazolid A (selected signals) (600 $\left.\mathrm{MHz}, \mathrm{CD}_{3} \mathrm{OD}\right)$

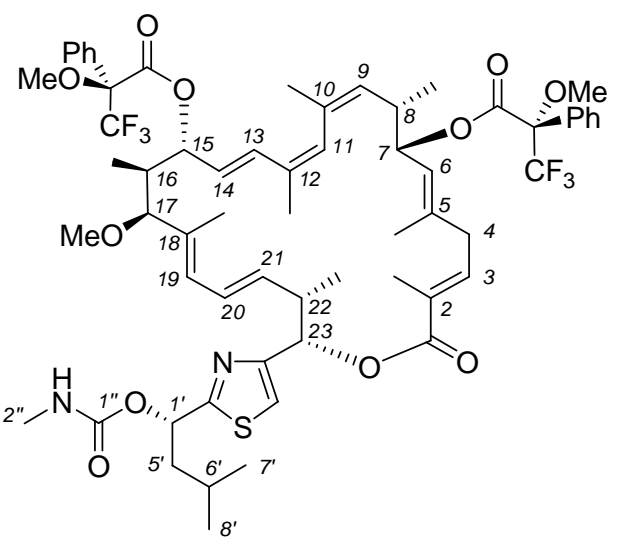

Bis-(S)-Mosher ester of archazolid A

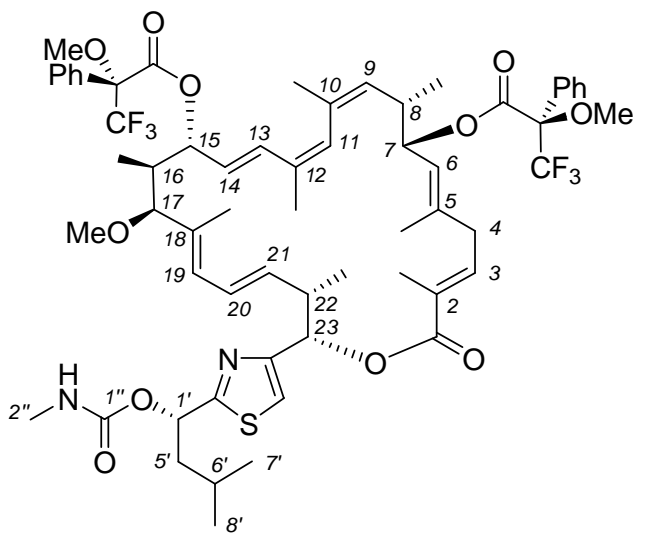

Bis-(R)-Mosher ester of archazolid A

\begin{tabular}{|c|l|l|l|}
\hline $\mathbf{H}$ & $\begin{array}{c}\boldsymbol{\delta} \text { [ppm], S-Mosher } \\
\text { ester of archazolid A }\end{array}$ & $\begin{array}{c}\boldsymbol{\delta} \text { [ppm], R-Mosher } \\
\text { ester of archazolid A }\end{array}$ & $\boldsymbol{\Delta} \boldsymbol{\delta}_{\mathbf{S}} \boldsymbol{\delta}_{\mathbf{S}}-\boldsymbol{\delta}_{\mathbf{R}}$ \\
\hline 6 & 5.25 & 5.07 & +0.18 \\
\hline 7 & 5.43 & 5.41 & +0.02 \\
\hline 9 & 5.13 & 5.29 & -0.16 \\
\hline 11 & 5.41 & 5.69 & -0.28 \\
\hline 13 & 6.51 & & -0.02 \\
\hline 14 & 5.75 & 5.61 & +0.14 \\
\hline 15 & 5.78 & 5.71 & +0.07 \\
\hline 17 & 2.96 & 3.12 & -0.16 \\
\hline 19 & 5.81 & 5.84 & -0.03 \\
\hline 20 & 5.76 & 6.01 & -0.25 \\
\hline 21 & 5.49 & 5.51 & -0.02 \\
\hline Me-2 & 1.91 & 1.90 & +0.01 \\
\hline Me-5 & 1.89 & 1.90 & -0.01 \\
\hline Me-8 & 0.99 & 1.01 & -0.02 \\
\hline Me-10 & 1.57 & 1.80 & -0.23 \\
\hline Me-12 & 1.83 & 1.80 & +0.03 \\
\hline Me-16 & 0.72 & 0.79 & -0.07 \\
\hline OMe-17 & 3.07 & 3.10 & -0.03 \\
\hline Me-18 & 1.52 & 1.58 & -0.06 \\
\hline Me-22 & 1.12 & 1.12 & 0 \\
\hline
\end{tabular}


Bis-TBS-ether of archazolid A (6)

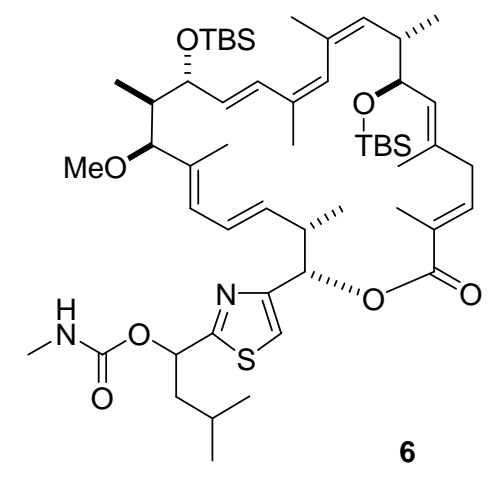

To a stirred solution of archazolid A (1, $37.0 \mathrm{mg}, 0.050 \mathrm{mmol})$ in DCM (1.5 mL) was added pyridine $(0.75 \mathrm{~mL}), \mathrm{DMAP}(3.0 \mathrm{mg}, 0.025 \mathrm{mmol})$ and TBSCl $(160 \mathrm{mg}, 1.06 \mathrm{mmol})$ at room temperature. After $8 \mathrm{~d}$, the mixture was filtered through a short pad of silica gel $(1 \times 5 \mathrm{~cm})$ with DCM and evaporated in vacuo. Silica gel chromatography (hexanes/EtOAc, gradient elution, 10:1 to $2: 1)$ afforded $6(33.8 \mathrm{mg}, 0.035 \mathrm{mmol}, 70 \%)$ as a colorless liquid. $\mathrm{R}_{f}=0.71$ (hexanes/EtOAc 2:1); $[\alpha]^{23}{ }_{\mathrm{D}}=-58.7\left(c=1, \mathrm{CHCl}_{3}\right) ;{ }^{1} \mathrm{H}-\mathrm{NMR}\left(600 \mathrm{MHz}, \mathrm{CDCl}_{3}\right) \delta 6.91(\mathrm{~s}, 1$ H) $6.76(\mathrm{t}, J=7.0 \mathrm{~Hz}, 1 \mathrm{H}) 6.28(\mathrm{~d}, J=15.9 \mathrm{~Hz}, 1 \mathrm{H}) 6.04(\mathrm{~d}, J=3.8 \mathrm{~Hz}, 1 \mathrm{H}) 6.01-6.07$ (m, 1 H) $5.86(\mathrm{dd}, J=14.7,11.0 \mathrm{~Hz}, 1 \mathrm{H}) 5.77(\mathrm{~d}, J=11.0 \mathrm{~Hz}, 1 \mathrm{H}) 5.69(\mathrm{dd}, J=16.1,7.7 \mathrm{~Hz}, 1 \mathrm{H})$ $5.65(\mathrm{~s}, 1 \mathrm{H}) 5.41(\mathrm{dd}, J=14.9,8.5 \mathrm{~Hz}, 1 \mathrm{H}) 5.12(\mathrm{~d}, J=9.1 \mathrm{~Hz}, 1 \mathrm{H}) 5.03(\mathrm{~d}, J=10.2 \mathrm{~Hz}, 1 \mathrm{H})$ 4.74 (d, J=4.5 Hz, 1 H) 4.40 (br. s, 1 H) 3.92 (t, J=8.9 Hz, 1 H) 3.12 (d, J=9.4 Hz, 1 H) 3.03 $3.09(\mathrm{~m}, 1 \mathrm{H}) 3.02(\mathrm{~s}, 3 \mathrm{H}) 2.91(\mathrm{dd}, J=14.7,8.7 \mathrm{~Hz}, 1 \mathrm{H}) 2.84(\mathrm{dd}, J=15.1,6.4 \mathrm{~Hz}, 1 \mathrm{H}) 2.80$ $(\mathrm{d}, J=4.5 \mathrm{~Hz}, 3 \mathrm{H}) 2.20(\mathrm{ddq}, J=9.1,7.0,6.8 \mathrm{~Hz}, 1 \mathrm{H}) 1.87-1.94(\mathrm{~m}, 1 \mathrm{H}) 1.85(\mathrm{~s}, 3 \mathrm{H}) 1.84$ $(\mathrm{s}, 3 \mathrm{H}) 1.79-1.83(\mathrm{~m}, 1 \mathrm{H}) 1.72(\mathrm{~s}, 1 \mathrm{H}) 1.72(\mathrm{~s}, 3 \mathrm{H}) 1.63(\mathrm{~s}, 3 \mathrm{H}) 1.53(\mathrm{~s}, 3 \mathrm{H}) 1.49$ - 1.56 $(\mathrm{m}, 1 \mathrm{H}) 1.11(\mathrm{~d}, J=6.8 \mathrm{~Hz}, 3 \mathrm{H}) 0.97(\mathrm{~d}, J=6.8 \mathrm{~Hz}, 3 \mathrm{H}) 0.95(\mathrm{~d}, J=6.8 \mathrm{~Hz}, 3 \mathrm{H}) 0.88(\mathrm{~s}, 9 \mathrm{H})$ $0.83(\mathrm{~s}, 9 \mathrm{H}) 0.80(\mathrm{~d}, J=6.8 \mathrm{~Hz}, 3 \mathrm{H}) 0.67(\mathrm{~d}, J=7.2 \mathrm{~Hz}, 3 \mathrm{H}) 0.09$ (s, $3 \mathrm{H}) 0.01$ (s, $3 \mathrm{H})-0.02$ $(\mathrm{s}, 3 \mathrm{H})-0.05(\mathrm{~s}, 3 \mathrm{H}) ;{ }^{13} \mathrm{C}-\mathrm{NMR}\left(100 \mathrm{MHz} \mathrm{CDCl}_{3}\right) \delta 170.7,166.9,155.9,155.4,140.5$, 136.4, 134.0, 132.9, 132.6, 132.2, 130.3, 130.1, 128.8, 127.6, 127.4, 126.6, 115.0, 86.5, 74.9, 73.7, 72.0, 55.3, 46.5, 44.5, 41.2, 40.9, 42.1 (2 C) 39.7, 27.6, 25.9, 25.724 .7 24.5, 22.9, 22.2 19.7, 18.2 18.0, 17.9, 16.8, 16.1 15.8, 12.4, 10.7 -3.6, -3.8, -4.3, -5.0; HRMS calculated for $\mathrm{C}_{54} \mathrm{H}_{90} \mathrm{~N}_{2} \mathrm{O}_{7} \mathrm{Si}_{2} \mathrm{~S}[M+\mathrm{H}]^{+}:$967.6086, found 967.6070. 
Ring Opening of the bis-TBS-ether of archazolid A (6)
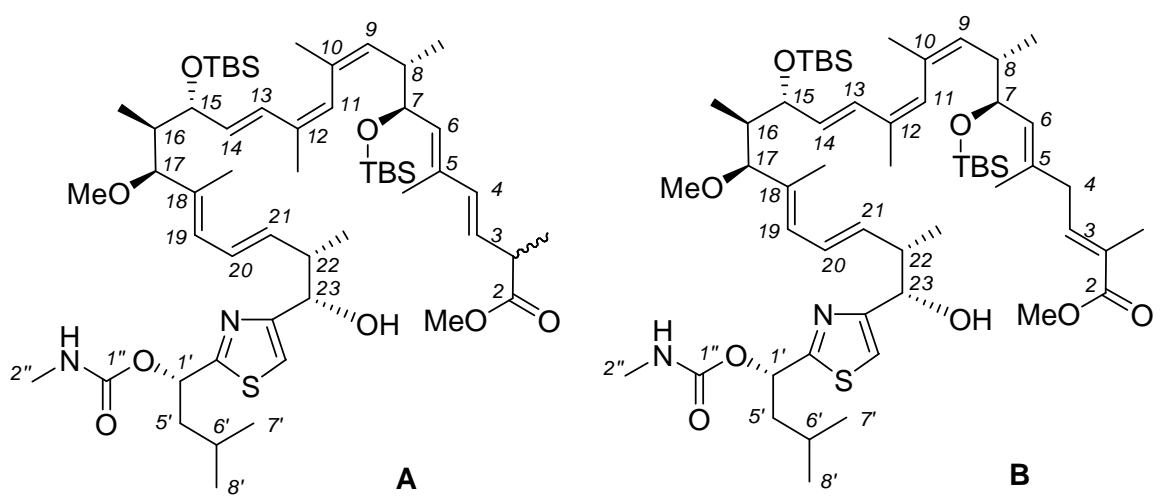

A solution of $6(8.3 \mathrm{mg}, 8.6 \mu \mathrm{mol})$ in diethyl ether $(500 \mu \mathrm{L})$ was treated under argon with a stock solution $(830 \mu \mathrm{L}$, Methanol) containing $\mathrm{LiBr}(7.4 \mathrm{mg}, 85.2 \mu \mathrm{mol})$ and $\mathrm{DBU}(1.3 \mu \mathrm{L}$, $1.33 \mathrm{mg}, 8.7 \mu \mathrm{mol})$. The resulting mixture was stirred at $60{ }^{\circ} \mathrm{C}$ for $44 \mathrm{~h}$. Evaporation of the solvent, flash chromatotography on silica gel (hexanes/EtOAc, 2:1) and HPLC separation on silica gel ( $n$-hexane/i-PrOH, 15:1, Nucleosil 100-7) gave $5.7 \mathrm{mg},(5.70 \mu \mathrm{mol}, 66 \%)$ of a mixture of the diastereomers $\mathbf{A}$ and $\mathbf{B}$ (ratio of 3:1, as determined by ${ }^{1} \mathrm{H}$ NMR), which were spectroscopically identical in the region H-9 to H-23 and H-1' to H-8' (see Table 4). $\mathrm{R}_{f}=0.34$ (hexanes/EtOAc 2:1). HRMS calculated for $\mathrm{C}_{55} \mathrm{H}_{95} \mathrm{~N}_{2} \mathrm{O}_{8} \mathrm{Si}_{2} \mathrm{~S}[M+\mathrm{H}]^{+}$: 999,6348, found 999,6352 .

Table 5. ${ }^{1} \mathrm{H}-\mathrm{NMR}$ data for isomers $\mathrm{A}$ and $\mathrm{B}\left(600 \mathrm{MHz}, \mathrm{CD}_{3} \mathrm{OD}\right)$

\begin{tabular}{|c|l|l|}
\hline $\mathbf{H}$ & \multicolumn{1}{|c|}{$\begin{array}{c}\boldsymbol{\delta}[\mathbf{p p m}], \text { mult., } \boldsymbol{J}[\mathbf{H z}] \\
\text { Isomer A }\end{array}$} & $\begin{array}{c}\boldsymbol{\delta} \text { [ppm], mult., } \boldsymbol{J}[\mathrm{Hz}] \\
\text { Isomer B }\end{array}$ \\
\hline 2 & $3.25, \mathrm{~m}$ & - \\
\hline 3 & $5.63, \mathrm{ddq}, 15.6 / 7.8 / 1.6$ & $6.65, \mathrm{td}, 7.7 / 1.1$ \\
\hline 4 & $6.13, \mathrm{~d}, 15.5$ & $2.85, \mathrm{~d}, 7.9$ \\
\hline 6 & $5.33, \mathrm{~d}, 9.1$ & $5.06, \mathrm{~d}, 10.2$ \\
\hline 7 & $4.23, \mathrm{dd}, 8.7 / 5.7$ & $4.10, \mathrm{dd}, 9.1 / 6.0$ \\
\hline 8 & $2.37, \mathrm{ddq}, 9.7 / 9.0 / 6.8$ & $2.29, \mathrm{ddq}, 9.8 / 9.5 / 6.8$ \\
\hline 9 & $5.02, \mathrm{~d}, 9.8$ & $5.02, \mathrm{~d}, 9.8$ \\
\hline 11 & $5.82, \mathrm{~s}$ & $5.82, \mathrm{~s}$ \\
\hline 13 & $6.34, \mathrm{~d}, 15.5$ & $6.34, \mathrm{~d}, 15.5$ \\
\hline 14 & $5.70, \mathrm{dd}, 16.2 / 6.0$ & $5.70, \mathrm{dd}, 16.2 / 6.0$ \\
\hline 15 & $4.65, \mathrm{dd}, 6.4 / 4.5$ & $4.65, \mathrm{dd}, 6.4 / 4.5$ \\
\hline 16 & $1.53, \mathrm{ddq}, 9.5 / 3.2 / 6.8$ & $1.53, \mathrm{ddq}, 9.5 / 3.2 / 6.8$ \\
\hline 17 & $3.25, \mathrm{~d}, 9.8$ & $3.25, \mathrm{~d}, 9.8$ \\
\hline 19 & $5.81, \mathrm{~d}, 9.8$ & $5.81, \mathrm{~d}, 9.8$ \\
\hline 20 & $6.10, \mathrm{dd}, 15.5 / 11.3$ & $6.10, \mathrm{dd}, 15.5 / 11.3$ \\
\hline 21 & $5.67, \mathrm{dd}, 15.8 / 7.2$ & $5.67, \mathrm{dd}, 15.8 / 7.2$ \\
\hline 22 & $2.70, \mathrm{ddq}, 7.0 / 4.5 / 6.8$ & $2.70, \mathrm{ddq}, 7.0 / 4.5 / 6.8$ \\
\hline 23 & $4.56, \mathrm{dd}, 5.4 / 4.5$ & $4.56, \mathrm{dd}, 5.4 / 4.5$ \\
\hline OH-23 & $5.30, \mathrm{~d}, 5.4$ & $5.30, \mathrm{~d}, 5.4$ \\
\hline Me-2 & $1.19, \mathrm{~d}, 6.8 / 1.20, \mathrm{~d}, 7.2$ & $1.78, \mathrm{~s}$ \\
\hline
\end{tabular}




\begin{tabular}{|c|l|l|}
\hline $\mathrm{Me}-5$ & $1.65, \mathrm{~s}$ & $1.58, \mathrm{~s}$ \\
\hline $\mathrm{Me}-8$ & $0.82, \mathrm{~d}, 6.8$ & $0.82, \mathrm{~d}, 6.8$ \\
\hline $\mathrm{Me}-10$ & $1.73, \mathrm{~s}$ & $1.73, \mathrm{~s}$ \\
\hline $\mathrm{Me}-12$ & $1.80, \mathrm{~s}$ & $1.80, \mathrm{~s}$ \\
\hline $\mathrm{Me}-16$ & $0.54, \mathrm{~d}, 6.8$ & $0.54, \mathrm{~d}, 6.8$ \\
\hline OMe-17 & $3.00, \mathrm{~s}$ & $3.00, \mathrm{~s}$ \\
\hline $\mathrm{Me}-18$ & $1.46, \mathrm{~s}$ & $1.46, \mathrm{~s}$ \\
\hline $\mathrm{Me}-22$ & $0.94, \mathrm{~d}, 6.8$ & $0.94, \mathrm{~d}, 6.8$ \\
\hline $1^{\prime}$ & $5.86, \mathrm{dd}, 8.3 / 5.7$ & $5.86, \mathrm{dd}, 8.3 / 5.7$ \\
\hline $4^{\prime}$ & $7.30, \mathrm{~s}$ & $7.30, \mathrm{~s}$ \\
\hline $5^{\prime}$ & $1.76, \mathrm{~m}$ & $1.76, \mathrm{~m}$ \\
\hline $6^{\prime}$ & $1.63, \mathrm{~m}$ & $1.63, \mathrm{~m}$ \\
\hline $7^{\prime}$ & $0.91, \mathrm{~d}, 6.4$ & $0.91, \mathrm{~d}, 6.4$ \\
\hline $8^{\prime}$ & $0.91, \mathrm{~d}, 6.4$ & $0.91, \mathrm{~d}, 6.4$ \\
\hline $2^{\prime \prime}$ & $2.57, \mathrm{~d}, 4.5$ & $2.57, \mathrm{~d}, 4.5$ \\
\hline $\mathrm{NH}^{\prime}$ & $7.27, \mathrm{q}, 4.5$ & $7.27, \mathrm{q}, 4.5$ \\
\hline $\mathrm{CO}_{2} \mathrm{Me}$ & $3.61, \mathrm{~s}$ & $3.66, \mathrm{~s}$ \\
\hline TBS $^{\prime}$ & $0.02, \mathrm{~s}$ & $0.02, \mathrm{~s}$ \\
\hline TBS $^{\prime}$ & $0.06, \mathrm{~s}$ & $0.06, \mathrm{~s}$ \\
\hline TBS & $0.90, \mathrm{~s}$ & $0.90, \mathrm{~s}$ \\
\hline TBS' $^{\prime}$ & $0.04, \mathrm{~s}$ & $0.04, \mathrm{~s}$ \\
\hline TBS' & $0.08, \mathrm{~s}$ & $0.08, \mathrm{~s}$ \\
\hline TBS' & $0.94, \mathrm{~s}$ & $0.94, \mathrm{~s}$ \\
\hline
\end{tabular}

Table 6. Selected ${ }^{1} \mathrm{H}$-NMR data for the Mosher esters derivatives $8 \mathrm{a}$ and $8 \mathrm{~b}(600 \mathrm{MHz}$, $\mathrm{CD}_{3} \mathrm{OD}$ )

\begin{tabular}{|l|l|l|l|}
\hline \multicolumn{1}{|c|}{$\mathbf{H}$} & $\begin{array}{c}\delta[\mathrm{ppm}], \\
\text { S-Mosher ester 8a }\end{array}$ & $\begin{array}{c}\delta[\mathrm{ppm}], \\
\text { R-Mosher ester 8 b }\end{array}$ & $\Delta \delta=\delta_{\mathrm{S}}-\delta_{\mathrm{R}}$ \\
\hline 19 & 5.77 & 5.81 & -0.04 \\
\hline 20 & 6.06 & 6.09 & -0.03 \\
\hline 21 & 5.59 & 5.68 & -0.09 \\
\hline 22 & 2.58 & 2.72 & -0.14 \\
\hline $\mathrm{Me}-22$ & 0.84 & 0.96 & -0.12 \\
\hline $1^{\prime}$ & 5.86 & 5.87 & +0.01 \\
\hline $4^{\prime}$ & 7.09 & 7.00 & +0.09 \\
\hline $7^{\prime}$ & 0.95 & 0.92 & +0.03 \\
\hline $8^{\prime}$ & 0.95 & 0.92 & +0.03 \\
\hline
\end{tabular}




\section{Cleavage of the Carbamate: Compound 10}

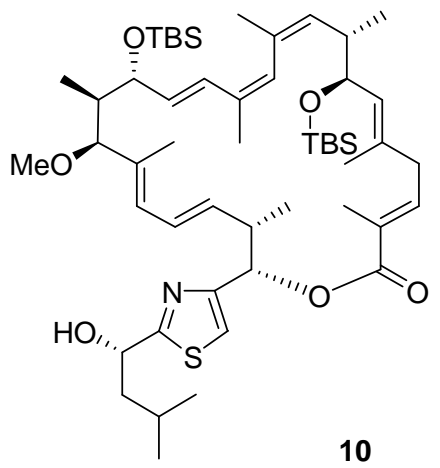

To a stirred solution of $6(5.6 \mathrm{mg}, 5.8 \mu \mathrm{mol})$ in toluene $(1 \mathrm{~mL})$ was added triethylamine $(0.3 \mathrm{~mL}, 2.2 \mathrm{mmol})$ and a solution of $\mathrm{HSiCl}_{3}(0.1 \mathrm{~mL}, 1.0 \mathrm{mmol})$ in toluene $(1 \mathrm{~mL})$ at room temperature. After $6 \mathrm{~h}$, the reaction was diluted with MTBE $(10 \mathrm{~mL})$ and quenched with $1 \mathrm{M}$ aqueous $\mathrm{NH}_{4} \mathrm{Cl}(5 \mathrm{~mL})$. The organic layer was separated and the aqueous layer was extracted with MTBE (3x $5 \mathrm{~mL}$ ). The combined organics were washed with $1 \mathrm{M}$ aqueous $\mathrm{NH}_{4} \mathrm{Cl}$ (5 mL), dried over $\mathrm{Na}_{2} \mathrm{SO}_{4}$, filtered and evaporated in vacuo. HPLC separation on silica gel (n-hexane/i-PrOH, 50:1, Nucleosil 100-7) afforded alcohol 10 (4.8 mg, $5.4 \mu \mathrm{mol}, 91 \%)$ as a colorless liquid. $\mathrm{R}_{f}=0.54$ (hexanes/EtOAc 4:1); $[\alpha]^{23}{ }_{\mathrm{D}}=-36.7\left(c=0.3, \mathrm{CHCl}_{3}\right) ;{ }^{1} \mathrm{H}-\mathrm{NMR}$ $\left(600 \mathrm{MHz}, \mathrm{CDCl}_{3}\right) \delta 6.97(\mathrm{~s}, 1 \mathrm{H}) 6.77(\mathrm{ddq}, J=8.3,6.8,1.5 \mathrm{~Hz}, 1 \mathrm{H}) 6.28(\mathrm{~d}, J=16.2 \mathrm{~Hz}, 1 \mathrm{H})$ $6.00(\mathrm{~d}, J=4.9 \mathrm{~Hz}, 1 \mathrm{H}) 5.95$ (dd, $J=15.1,11.0 \mathrm{~Hz}, 1 \mathrm{H}) 5.79$ (d, $J=10.6 \mathrm{~Hz}, 1 \mathrm{H}) 5.70$ (dd, $J=16.2,7.9 \mathrm{~Hz}, 1 \mathrm{H}) 5.66(\mathrm{~s}, 1 \mathrm{H}) 5.46(\mathrm{dd}, J=15.3,8.1 \mathrm{~Hz}, 1 \mathrm{H}) 5.12(\mathrm{~d}, J=9.1 \mathrm{~Hz}, 1 \mathrm{H}) 5.03$ (dd, $J=9.8,1.1 \mathrm{~Hz}, 1 \mathrm{H}) 4.99-5.04(\mathrm{~m}, 1 \mathrm{H}) 4.38-4.45(\mathrm{~m}, 1 \mathrm{H}) 3.92(\mathrm{t}, J=8.9 \mathrm{~Hz}, 1 \mathrm{H}) 3.14$ (d, J=9.4 Hz, 1 H) 3.04 (s, 3 H) $3.02-3.09$ (m, 1 H) 2.90 (dd, J=15.1, 7.9 Hz, 1 H) 2.82 - 2.87 (m, J=15.1, 6.4 Hz, $1 \mathrm{H}) 2.12-2.25(\mathrm{~m}, 1 \mathrm{H}) 1.98-2.05(\mathrm{~m}, 1 \mathrm{H}) 1.87$ - $1.94(\mathrm{~m}, 1 \mathrm{H}) 1.85$ (s, 3 H) 1.84 (d, J=1.1 Hz, 3 H) 1.78 (ddd, J=14.1, 9.0, 5.3 Hz, 1 H) 1.72 (s, 3 H) 1.69 - 1.75 $(\mathrm{m}, 1 \mathrm{H}) 1.64(\mathrm{~s}, 1 \mathrm{H}) 1.56(\mathrm{~d}, J=0.8 \mathrm{~Hz}, 3 \mathrm{H}) 1.52-1.61(\mathrm{~m}, 3 \mathrm{H}) 1.10(\mathrm{~d}, J=7.2 \mathrm{~Hz}, 3 \mathrm{H})$ $1.00(\mathrm{~d}, J=6.4 \mathrm{~Hz}, 3 \mathrm{H}) 0.98(\mathrm{~d}, J=6.8 \mathrm{~Hz}, 3 \mathrm{H}) 0.88(\mathrm{~s}, 9 \mathrm{H}) 0.84(\mathrm{~s}, 9 \mathrm{H}) 0.80(\mathrm{~d}, J=6.8 \mathrm{~Hz}, 3$ H) $0.68(\mathrm{~d}, J=7.2 \mathrm{~Hz}, 3 \mathrm{H}) 0.02(\mathrm{~s}, 3 \mathrm{H})-0.01(\mathrm{~s}, 3 \mathrm{H})-0.02(\mathrm{~s}, 3 \mathrm{H})-0.05(\mathrm{~s}, 3 \mathrm{H}) ;{ }^{13} \mathrm{C}-\mathrm{NMR}$ $\left(100 \mathrm{MHz}, \mathrm{CDCl}_{3}\right) \delta 174.5,167.0,154.7,140.4,136.4,134.1,133.0,132.8,132.6,132.2$, $132.1,130.3,130.1,128.8,127.6,127.3,126.6,115.3,86.69,77.3,77.2,76.7,76.4,73.7$, 72.8, 70.4, 55.3, 49.4, 47.5, 41.1, 40.9, 39.7, 31.9, 25.9, 25.8, 24.7, 24.5, 23.3, 22.7, 21.9, $19.8,18.2,18.1,18.0,16.8,16.1,12.4,10.7,-3.8,-4.3,-4.8,-5.0$; HRMS calculated for $\mathrm{C}_{52} \mathrm{H}_{87} \mathrm{NO}_{6} \mathrm{Si}_{2} \mathrm{~S}[M]^{+}:$909.5793, found 909.5787. 
Table 7. Selected ${ }^{1} \mathrm{H}-\mathrm{NMR}$ data for the Mosher esters derivatives $11 \mathrm{a}$ and $11 \mathrm{~b}$ (600 $\left.\mathrm{MHz}, \mathrm{CD}_{3} \mathrm{OD}\right)$

\begin{tabular}{|c|c|c|c|}
\hline $\mathbf{H}$ & $\begin{array}{c}\boldsymbol{\delta}[\mathbf{p p m}], \\
\text { S-Mosher ester 11a }\end{array}$ & $\begin{array}{c}\boldsymbol{\delta}[\mathbf{p p m}], \\
\text { R-Mosher ester 11b }\end{array}$ & $\mathbf{\Delta} \boldsymbol{\delta}=\boldsymbol{\delta}_{\mathbf{S}}-\boldsymbol{\delta}_{\mathbf{R}}$ \\
\hline $4^{\prime}$ & 7.02 & 6.95 & +0.07 \\
\hline $5^{\prime}$ & 1.62 & 1.77 & -0.15 \\
\hline $6^{\prime}$ & 1.54 & 1.64 & -0.10 \\
\hline $7^{\prime}$ & 0.91 & 0.95 & -0.04 \\
\hline $8^{\prime}$ & 0.91 & 0.95 & -0.04 \\
\hline
\end{tabular}


Table 8. Comparison of the ${ }^{1} \mathrm{H}-\mathrm{NMR}$ data for archazolid $\mathrm{A}$ and $\mathrm{B}\left(600 \mathrm{MHz}, \mathrm{CD}_{3} \mathrm{OD}\right)$
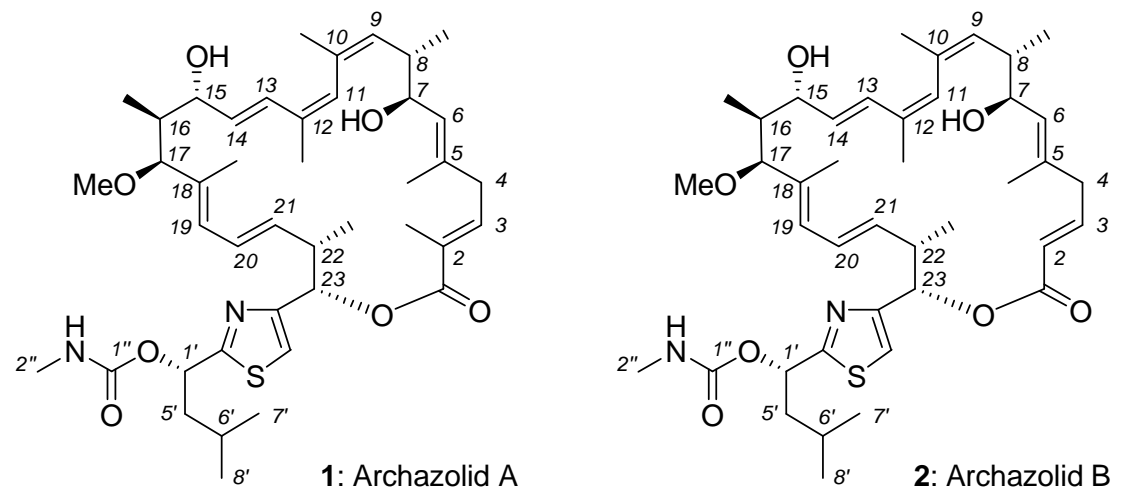

\begin{tabular}{|c|c|c|}
\hline $\mathbf{H}$ & $\begin{array}{c}\delta[\mathrm{ppm}], \text { mult., } J[\mathrm{~Hz}] \\
\text { archazolid A }\end{array}$ & $\begin{array}{c}\delta[\mathrm{ppm}], \text { mult., } J[\mathrm{~Hz}] \\
\text { archazolid B }\end{array}$ \\
\hline 2 & 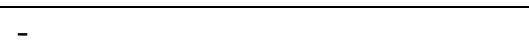 & $5.92, \mathrm{~d}, 15.7$ \\
\hline 3 & 6.82, ddd, $7.9 / 7.5 / 1.3$ & 6.94, ddd, $15.5 / 8.4 / 6.4$ \\
\hline $4 \mathrm{H}$ & $\begin{array}{l}2.91, \mathrm{dd}, 15.0 / 7.5 \\
3.03, \mathrm{dd}, 15.0 / 7.5\end{array}$ & $\begin{array}{l}2.91, \mathrm{dd}, 14.7 / 6.6 \\
2.96, \mathrm{dd}, 14.7 / 8.6\end{array}$ \\
\hline 6 & $5.21, \mathrm{dd}, 9.5 / 1.2$ & $5.18, \mathrm{dd}, 9.2 / 1.0$ \\
\hline 7 & $4.03, \mathrm{dd}, 9.4 / 9.3$ & $4.03, \mathrm{dd}, 9.2 / 9.2$ \\
\hline 8 & 2.30, ddq, $9.5 / 9.8 / 7.0$ & $2.30, \mathrm{ddq}, 9.5 / 9.5 / 7.0$ \\
\hline 9 & $5.27, \mathrm{~d}, 9.6$ & $5.28, \mathrm{~d}, 9.7$ \\
\hline 11 & $5.81, \mathrm{~d}, 1.0$ & $5.79, \mathrm{~d}, 0.6$ \\
\hline 13 & $6.56, \mathrm{dd}, 15.5 / 0.9$ & $6.60, \mathrm{~d}, 15.8$ \\
\hline 14 & $5.79, \mathrm{dd}, 15.5 / 6.0$ & $5.77, \mathrm{dd}, 16.2 / 5.6$ \\
\hline 15 & $4.31, \mathrm{dd}, 6.4 / 3.2$ & $4.38, \mathrm{dd}, 5.6 / 3.2$ \\
\hline 16 & $1.80, \mathrm{ddq}, 9.0 / 3.2 / 7.0$ & $1.79, \mathrm{~m}$ \\
\hline 17 & $3.40, \mathrm{~d}, 9.0$ & $3.47, \mathrm{~d}, 9.2$ \\
\hline 19 & $5.87, \mathrm{dd}, 10.9 / 1.2$ & $5.90, \mathrm{~d}, 10.2$ \\
\hline 20 & $6.17, \mathrm{dd}, 15.5 / 10.9$ & 6.28, ddd, $15.3 / 10.7 / 1.0$ \\
\hline 21 & $5.63, \mathrm{dd}, 15.2 / 7.0$ & $5.75, \mathrm{dd}, 15.3 / 6.6$ \\
\hline 22 & $3.10, \mathrm{ddq}, 7.5 / 4.0 / 7.0$ & $3.09, \mathrm{ddq}, 6.6 / 4.6 / 6.8$ \\
\hline 23 & $5.97, \mathrm{~d}, 4.1$ & $5.90, \mathrm{~d}, 4.4$ \\
\hline Me-2 & $1.91, \mathrm{~d}, 1.2$ & - \\
\hline Me-5 & $1.73, \mathrm{~d}, 1.2$ & $1.74, \mathrm{~d}, 1.0$ \\
\hline Me-8 & $0.84, \mathrm{~d}, 7.0$ & $0.81, \mathrm{~d}, 6.6$ \\
\hline Me-10 & 1.80, brs & 1.79, brs \\
\hline $\mathrm{Me}-12$ & $1.93, \mathrm{~d}, 1.2$ & $1.93, \mathrm{~d}, 1.1$ \\
\hline Me-16 & $0.74, \mathrm{~d}, 7.0$ & $0.72, \mathrm{~d}, 7.1$ \\
\hline OMe-17 & $3.16, \mathrm{~s}$ & $3.19, \mathrm{~s}$ \\
\hline Me-18 & $1.64, \mathrm{~d}, 1.2$ & $1.67, \mathrm{~d}, 1.0$ \\
\hline $\mathrm{Me}-22$ & $1.15, \mathrm{~d}, 7.0$ & $1.11, \mathrm{~d}, 6.8$ \\
\hline $1^{\prime}$ & $6.04, \mathrm{dd}, 9.1 / 4.5$ & $6.03, \mathrm{dd}, 8.9 / 4.8$ \\
\hline $44^{\prime}$ & $7.21, \mathrm{~s}$ & $7.31, \mathrm{~s}$ \\
\hline $5^{\prime}$ & $1.92, \mathrm{~m}$ & $1.92, \mathrm{~m}$ \\
\hline $6^{\prime}$ & $1.77, \mathrm{~m}$ & $1.78, \mathrm{~m}$ \\
\hline $77^{\prime}$ & $1.01, \mathrm{~d}, 6.0$ & $1.02, \mathrm{~d}, 6.1$ \\
\hline $8^{\prime}$ & $1.02, \mathrm{~d}, 6.0$ & 1.01, d. 6.6 \\
\hline $2^{\prime \prime}$ & $2.75, \mathrm{~s}$ & $2.75, \mathrm{~s}$ \\
\hline
\end{tabular}


Table 9. Comparison of the ${ }^{13} \mathrm{C}$-NMR data for archazolid $\mathrm{A}$ and $\mathrm{B}$ (600 $\left.\mathrm{MHz}, \mathrm{CD}_{3} \mathrm{OD}\right)$
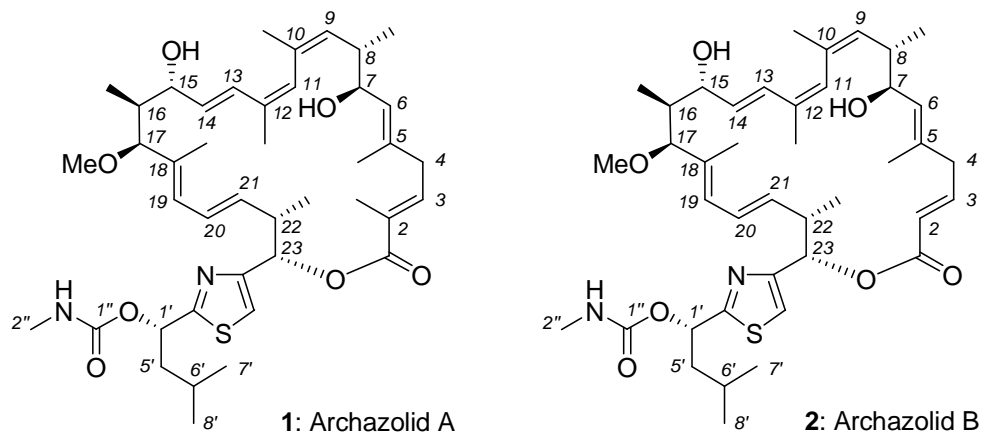

\begin{tabular}{|c|c|c|}
\hline C & $\delta[\mathrm{ppm}]$ archazolid A & $\delta$ [ppm] archazolid B \\
\hline 1 & 168.3 & 166.7 \\
\hline 2 & 129.7 & 123.3 \\
\hline 3 & 142.1 & 149.0 \\
\hline 4 & 40.6 & 44.0 \\
\hline 5 & 136.9 & 136.2 \\
\hline 6 & 130.4 & 130.7 \\
\hline 7 & 73.6 & 73.8 \\
\hline 8 & 41.6 & 41.5 \\
\hline 9 & 132.7 & 132.9 \\
\hline 10 & 134.8 & 134.6 \\
\hline 11 & 130.8 & 130.7 \\
\hline 12 & 133.6 & 133.4 \\
\hline 13 & 129.3 & 129.4 \\
\hline 14 & 133.6 & 133.5 \\
\hline 15 & 75.5 & 74.8 \\
\hline 16 & 44.5 & 44.0 \\
\hline 17 & 89.8 & 90.0 \\
\hline 18 & 135.7 & 135.6 \\
\hline 19 & 129.9 & 130.0 \\
\hline 20 & 127.6 & 127.3 \\
\hline 21 & 135.1 & 135.5 \\
\hline 22 & 42.0 & 41.3 \\
\hline 23 & 77.6 & 77.2 \\
\hline $\mathrm{Me}-2$ & 12.6 & - \\
\hline $\mathrm{Me}-5$ & 16.8 & 16.9 \\
\hline $\mathrm{Me}-8$ & 17.7 & 17.7 \\
\hline Me-10 & 24.7 & 24.7 \\
\hline Me-12 & 19.9 & 19.9 \\
\hline Me-16 & 12.6 & 12.3 \\
\hline OMe-17 & 56.2 & 56.4 \\
\hline Me-18 & 13.0 & 12.6 \\
\hline $\mathrm{Me}-22$ & 17.6 & 17.1 \\
\hline $1^{\prime}$ & 73.3 & 73.4 \\
\hline $2^{\prime}$ & 173.9 & 174.2 \\
\hline $3^{\prime}$ & 156.1 & 155.7 \\
\hline $4^{\prime}$ & 116.7 & 117.5 \\
\hline $5^{\prime}$ & 46.0 & 46.0 \\
\hline $6^{\prime}$ & 25.8 & 25.8 \\
\hline $7^{\prime}$ & 23.4 & 22.3 \\
\hline 81 & 22.4 & 23.4 \\
\hline 1" & 158.2 & 158.3 \\
\hline $2 "$ & 27.5 & 27.5 \\
\hline
\end{tabular}




\section{Copies of 1D and 2D NMR spectra}

1H NMR Spectrum of archazolid $\mathrm{A}(1)$ in $\mathrm{CD}_{3} \mathrm{OD}(600 \mathrm{MHz})$

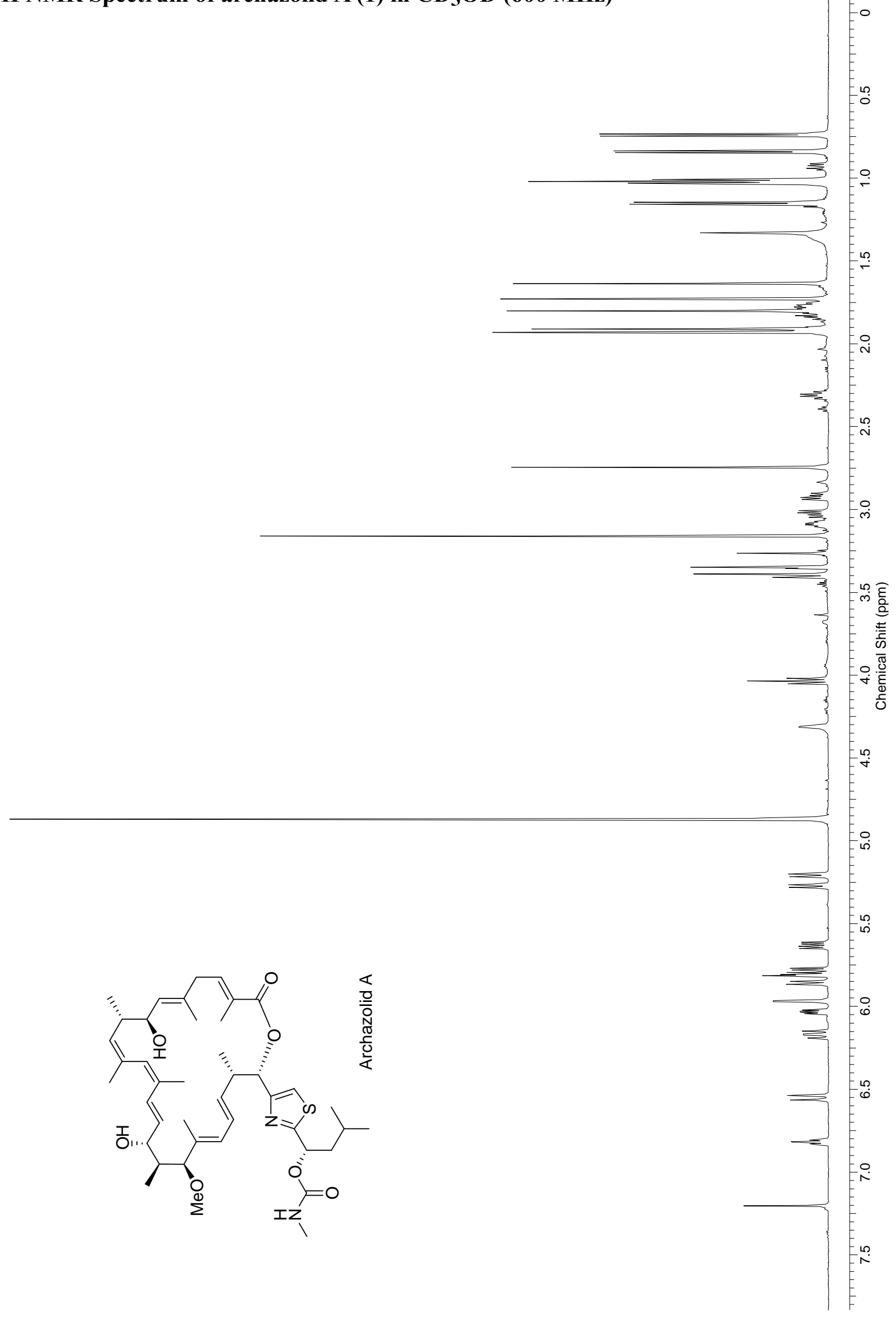


13C NMR Spectrum of archazolid A (1) in $\mathrm{CD}_{3} \mathrm{OD}(100 \mathrm{MHz})$

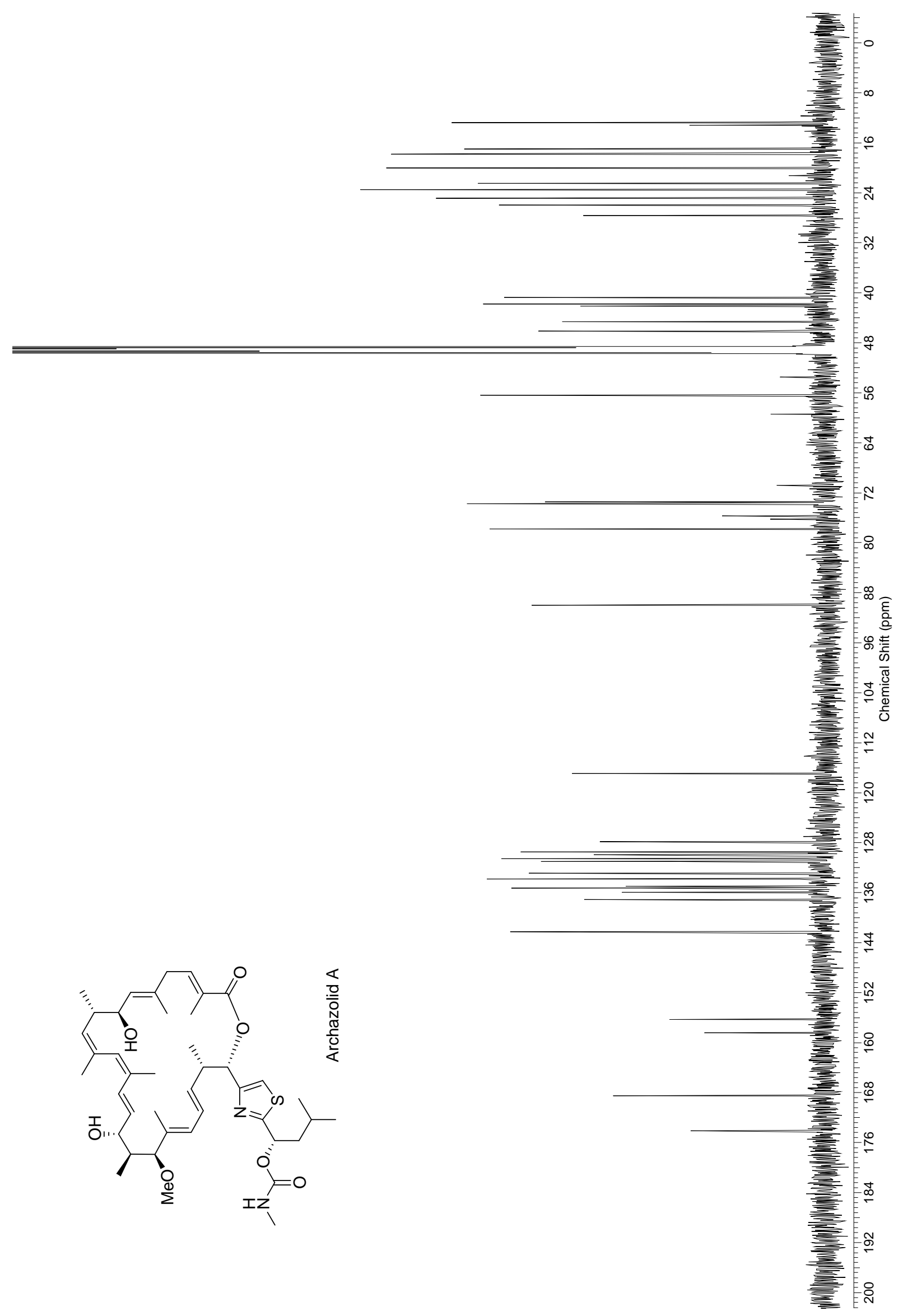




\section{ROESY Spectrum of Archazolid A (1) in $\mathrm{CD}_{3} \mathrm{OD}(600 \mathrm{MHz})$}

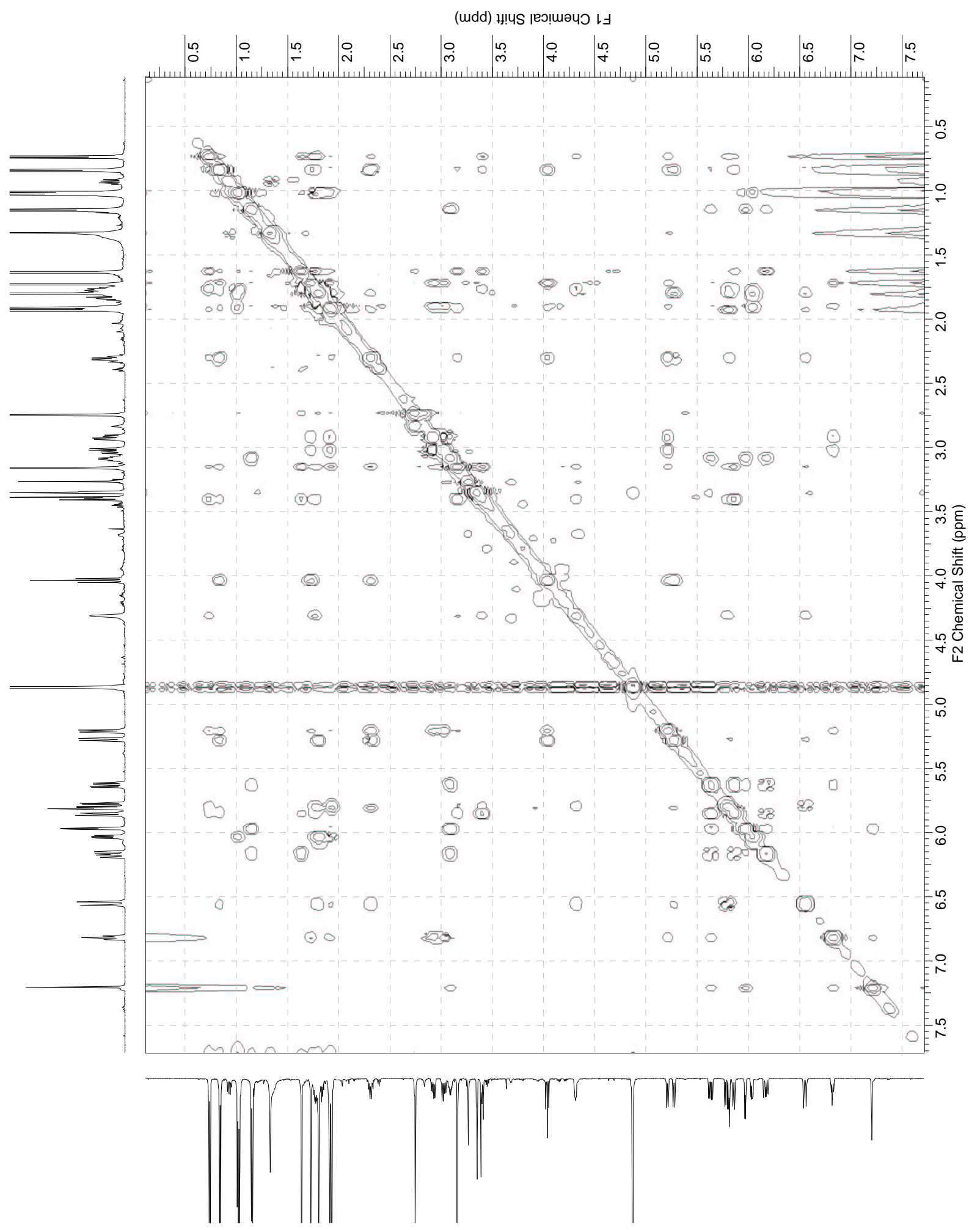


HSQC-HECADE Spectrum of archazolid A (1) in in DMSO-d 6 (600 MHz)

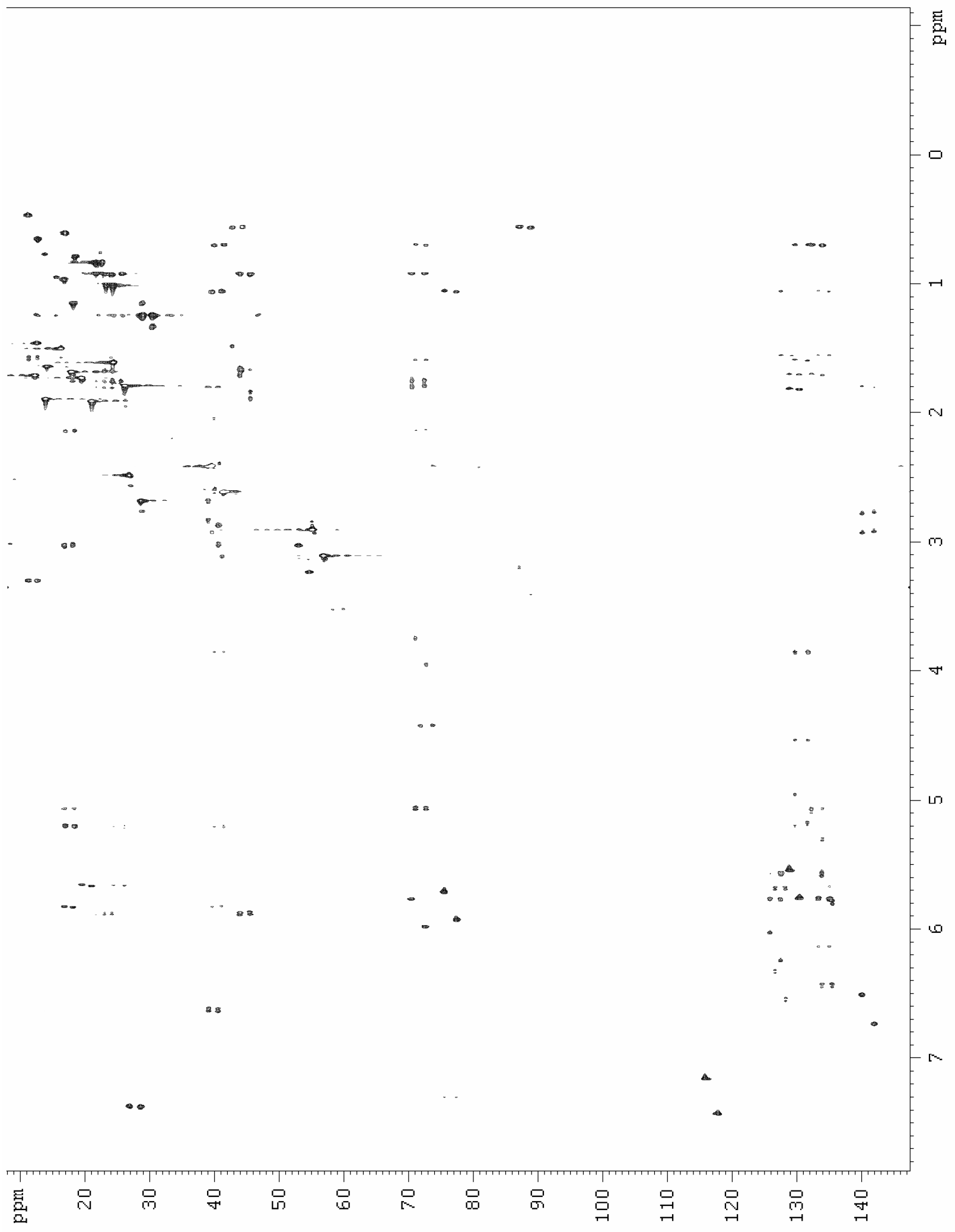


1H NMR Spectrum of compound 6 in $\mathrm{CDCl}_{3}(600 \mathrm{MHz})$

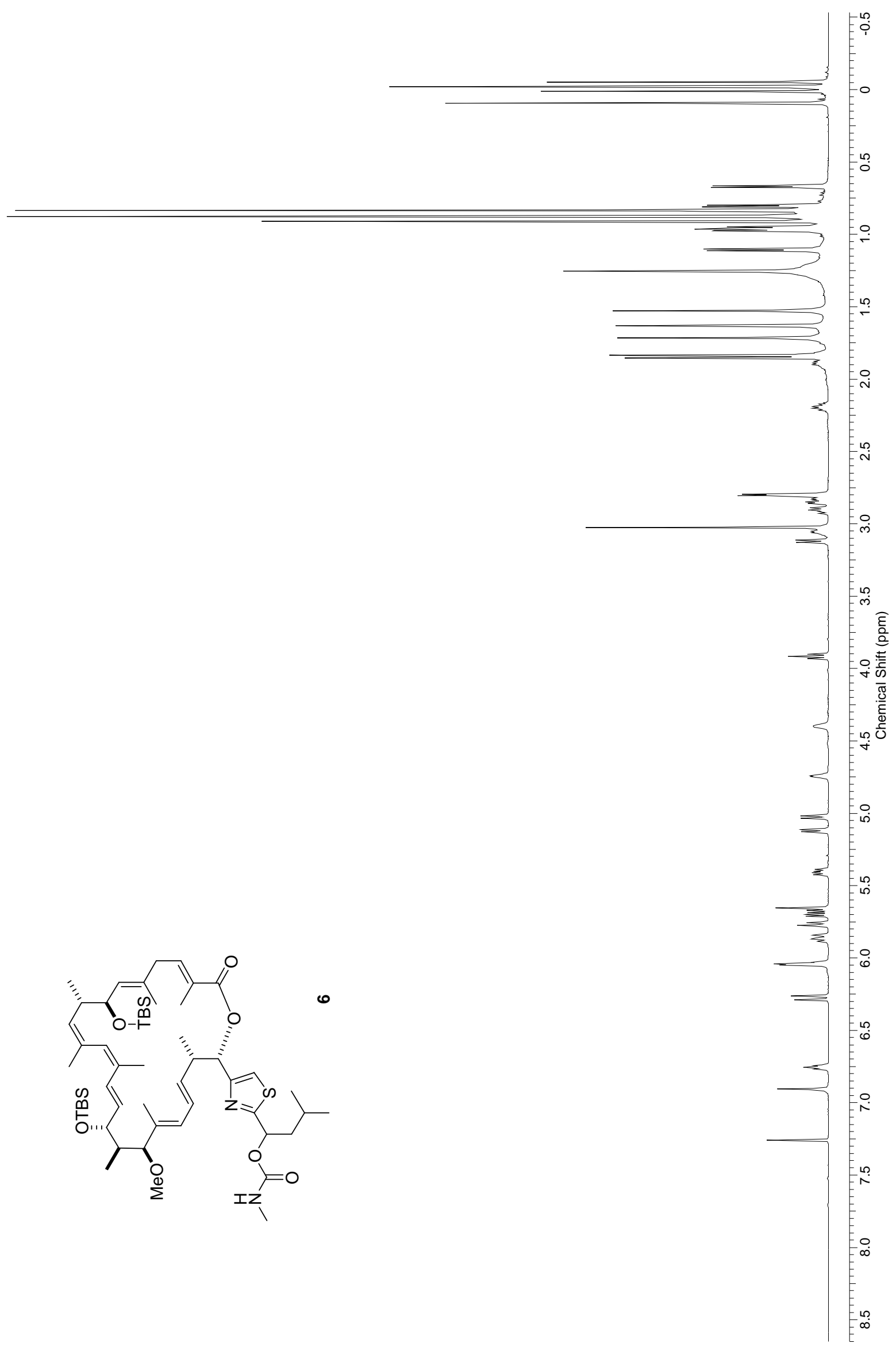


13C NMR Spectrum of compound 6 in $\mathrm{CDCl}_{3}(100 \mathrm{MHz})$

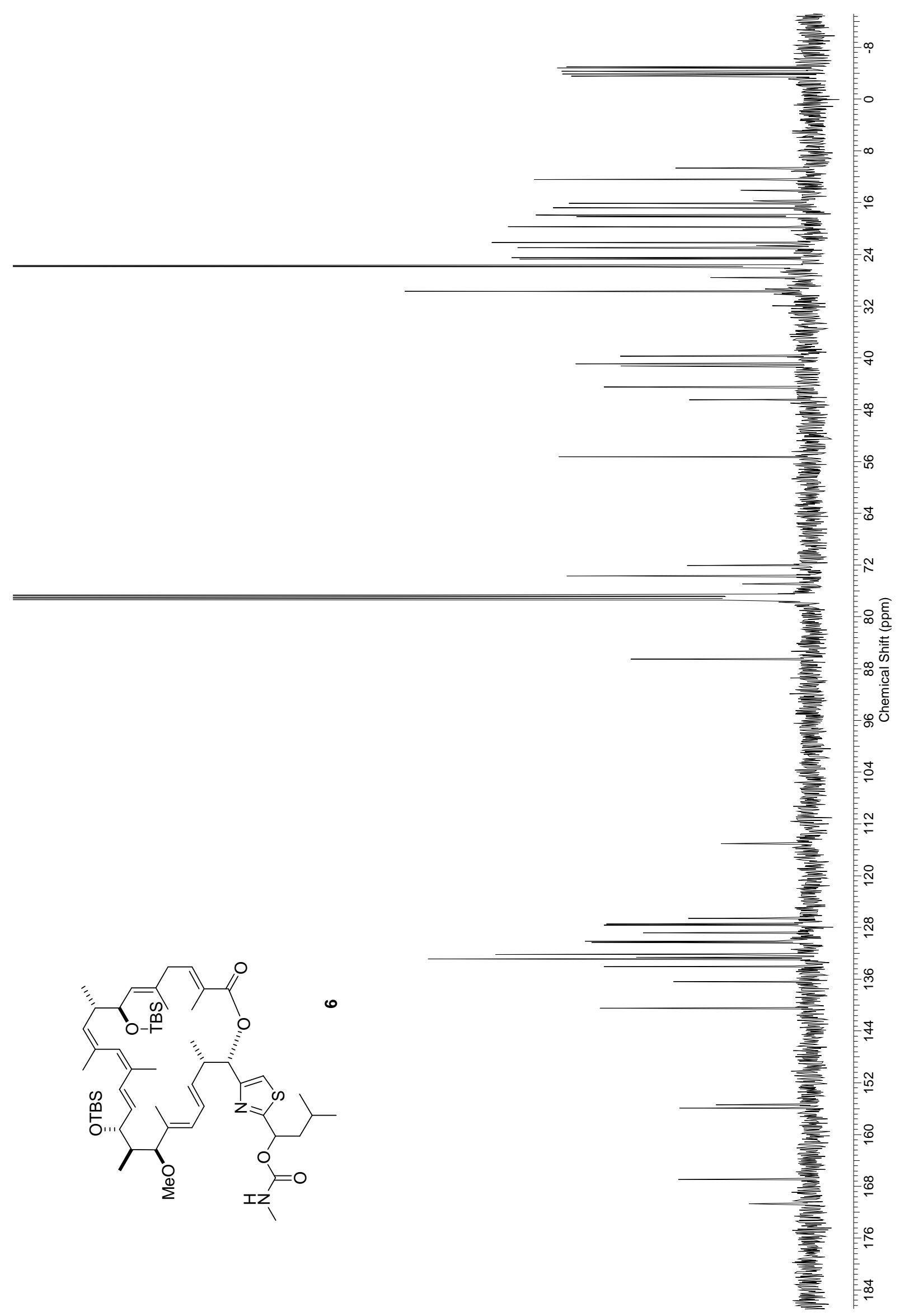


1H NMR Spectrum of compound 10 in $\mathrm{CDCl}_{3}(600 \mathrm{MHz})$

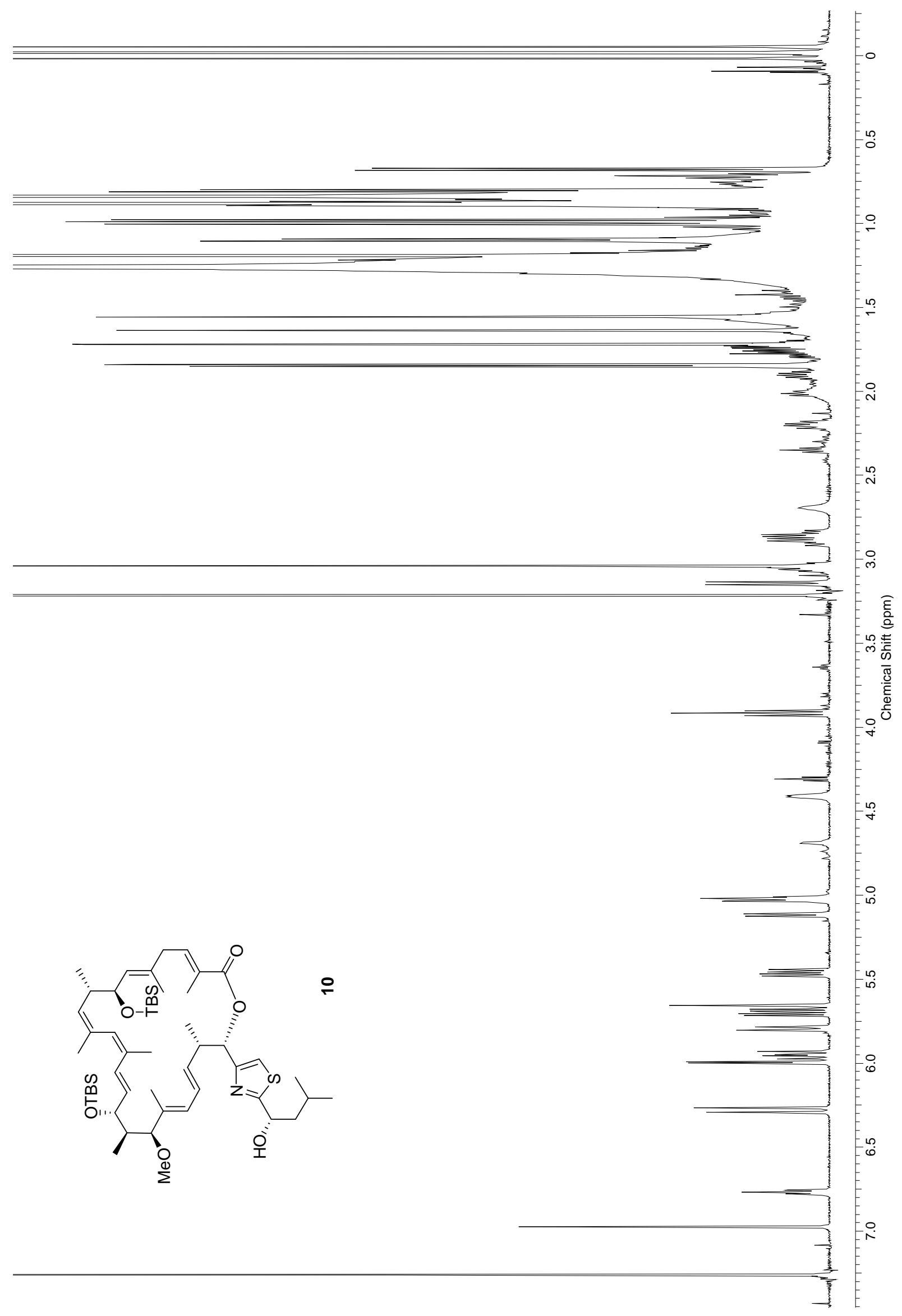


13C NMR Spectrum of compound 10 in $\mathrm{CDCl}_{3}(100 \mathrm{MHz})$

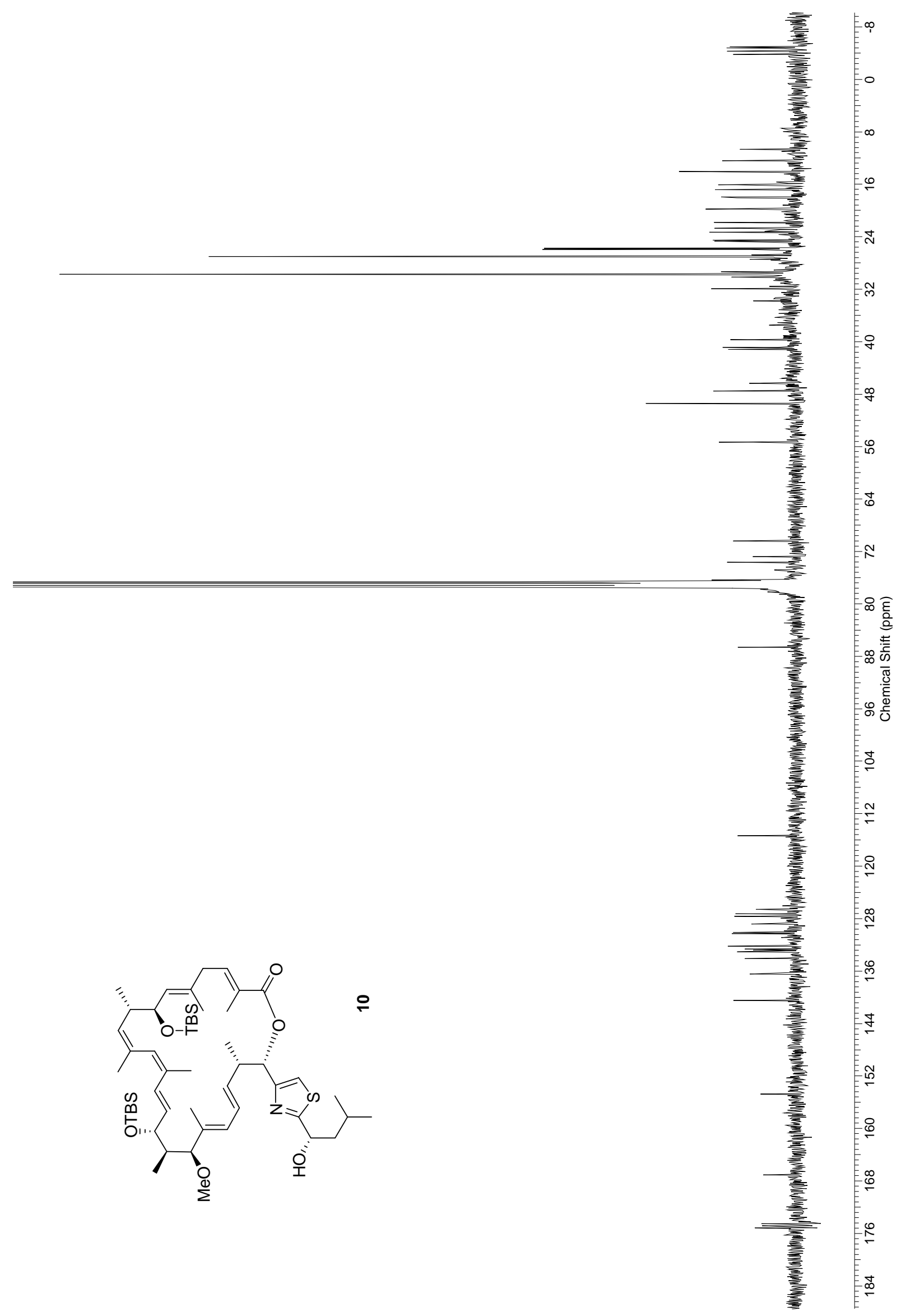


Overlay of the $1 \mathrm{H}$ spectram of archazolid $\mathrm{A}$ and $\mathrm{B}$ in $\mathrm{CD}_{3} \mathrm{OD}(600 \mathrm{MHz})$

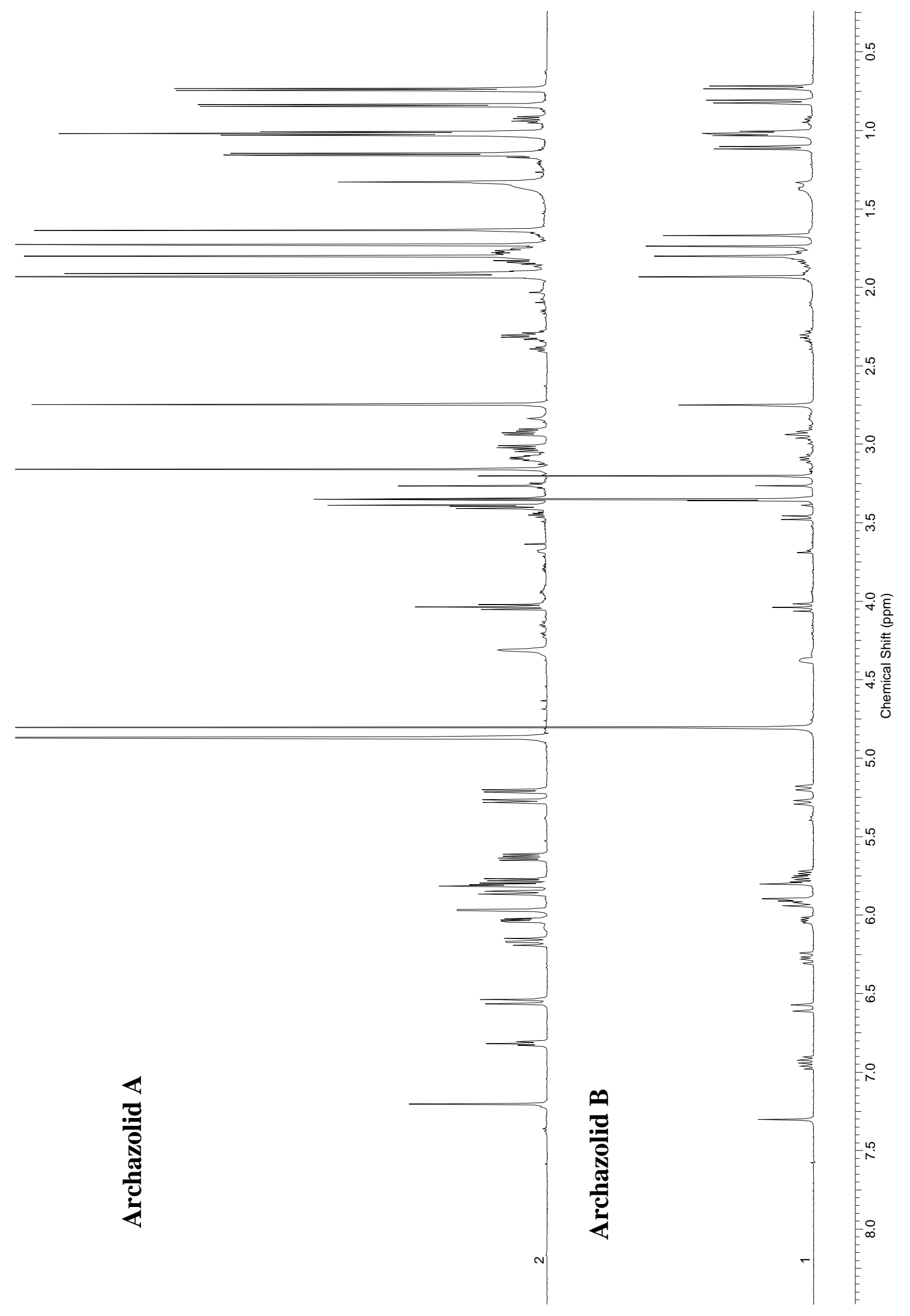




\section{Molecular Modeling}

Molecular modeling studies were performed on potential diastereomers of archazolid using Macromodel (version 8.5) and the MMFFs force field, together with the generalized Born/Surface area (GB/SA) water solvent model. Structures were subjected to a minimization procedure to the nearest local minimum prior to the generation of new local energy conformers by Monte Carlo searching (20,000 steps). All conformations within $50 \mathrm{KJ} \mathrm{mol}^{-1}$ were recorded. The normal set-up protocol was employed, with experiments sampling batches of 1000 to 2000 structures. The obtained low energy conformations were further refined by calculation of their AM 1 energies with Gaussian 03. 
Perspective drawing of the lowest energy conformation of archazolid A generated by Macromodel V 8.5

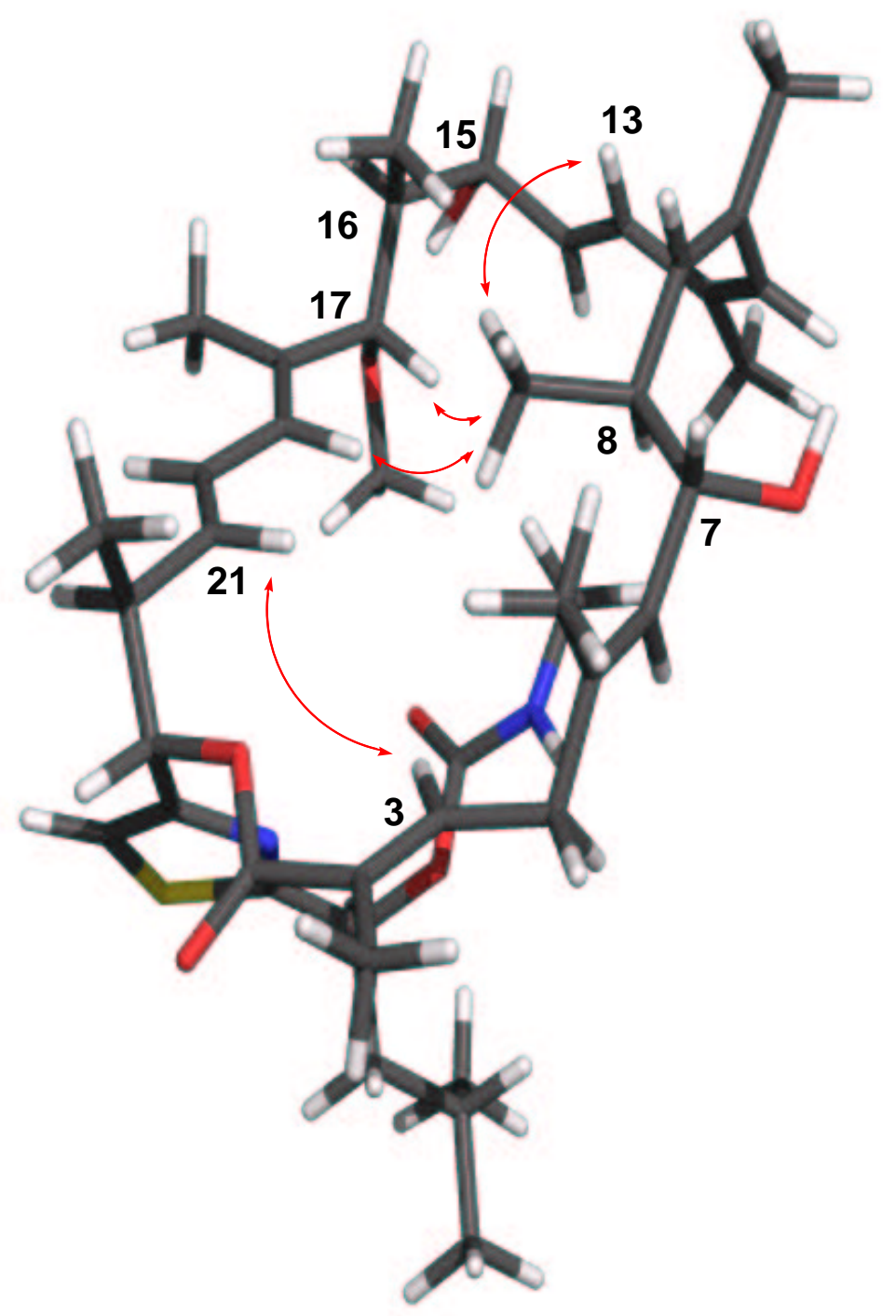

: selected transannular NOE-correlations

Lowest energy conformer (63.75 kcal) in MacroModel, Hartree-Fock energy: -0,3238206 hartree $(\mathrm{AM} \mathrm{1}), \mathrm{E}_{\mathrm{rel}}=0.00 \mathrm{kcal} \mathrm{mol}^{-1}$. 
Perspective Drawing of the 5A conformation of archazolid A (1) generated by Macromodel V 8.5

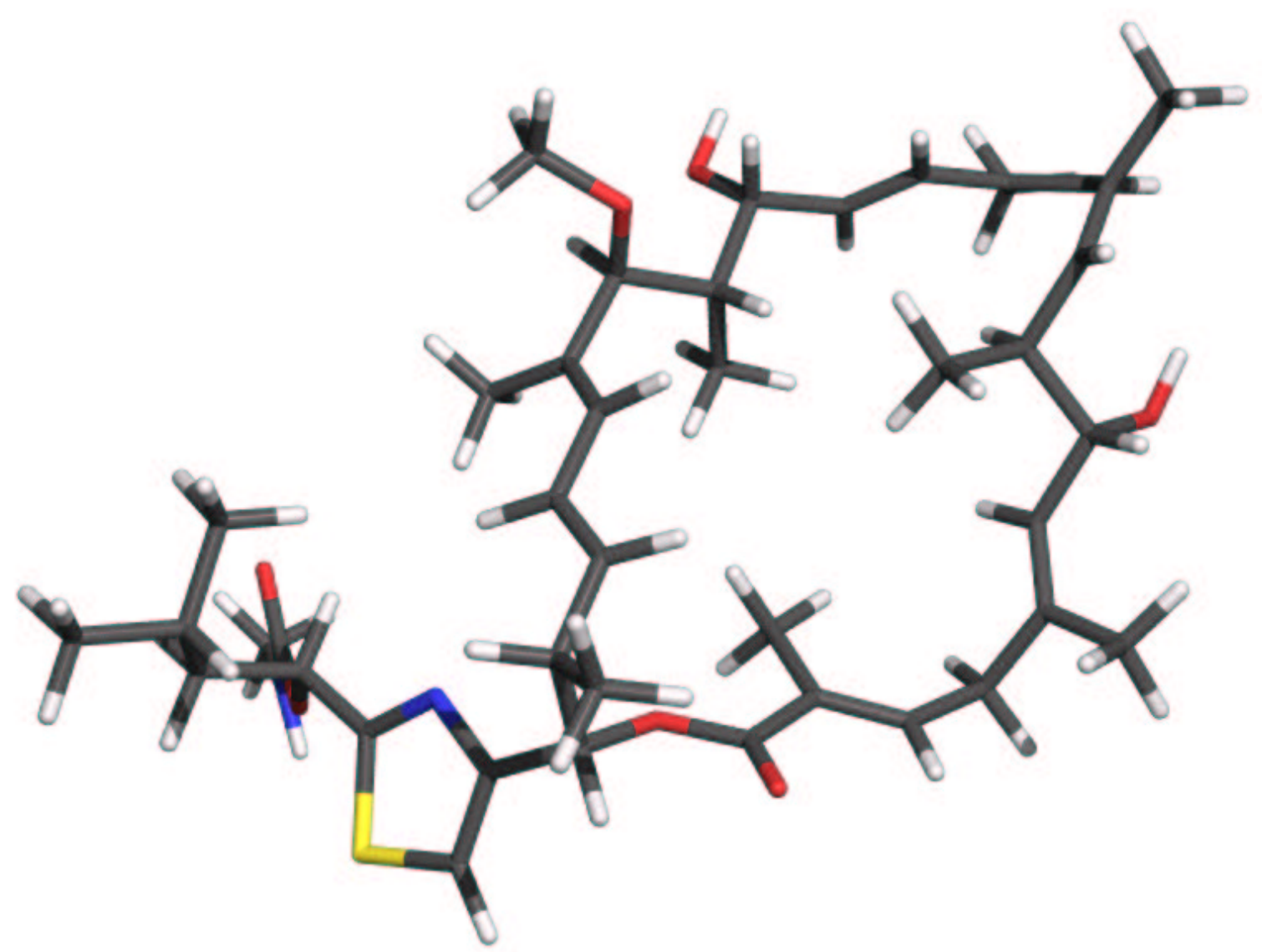

Lowest energy conformer $\mathbf{5 A}$ as obtained in macromodel using restrained dihedral angles and approximate $\mathrm{H}-\mathrm{H}$ distances obtained from ROESY experiments: $114.65 \mathrm{kcal} \mathrm{mol}^{-1}$ in MacroModel, Hartree-Fock energy: $-0,3155349$ hartree (AM 1), $\mathrm{E}_{\text {rel }}=5.20 \mathrm{kcal} \mathrm{mol}^{-1}$. 
Perspective drawing of the 5B conformation of archazolid A (1)

generated by Macromodel V 8.5

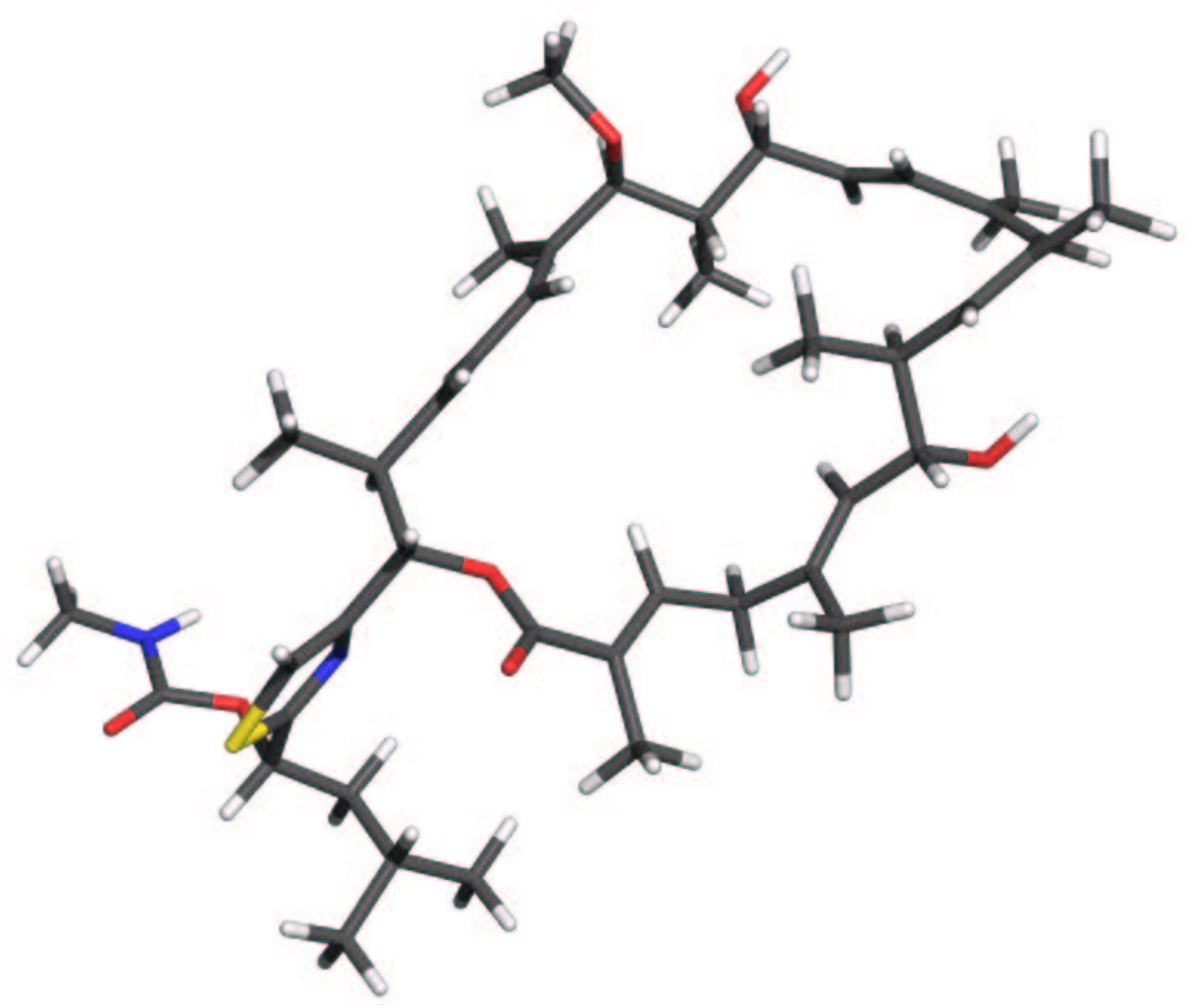

Lowest energy conformer 5B as obtained in macromodel using restrained dihedral angles and approximate $\mathrm{H}-\mathrm{H}$ distances obtained from ROESY experiments: $98.09 \mathrm{kcal} \mathrm{mol}^{-1}$ in MacroModel, Hartree-Fock energy: $-0,3206286$ hartree (AM 1), $\mathrm{E}_{\mathrm{rel}}=2.00 \mathrm{kcal} \mathrm{mol}^{-1}$. 
PDB output-file for the lowest energy conformation of archzolid A

\begin{tabular}{|c|c|c|c|c|c|c|c|c|c|}
\hline HEADER & \multicolumn{9}{|c|}{ PROTEIN } \\
\hline COMPND & \multicolumn{9}{|c|}{ SMALL MOLECULE } \\
\hline AUTHOR & \multicolumn{9}{|c|}{ GENERATED BY MOLEKEL } \\
\hline ATOM & 1 & $\mathrm{C}$ & unk & 1 & -2.806 & -1.543 & 3.818 & 1.00 & 0.00 \\
\hline ATOM & 2 & $\mathrm{C}$ & unk & 1 & -1.591 & -2.361 & 4.111 & 1.00 & 0.00 \\
\hline ATOM & 3 & $\mathrm{C}$ & unk & 1 & -0.563 & -2.339 & 3.237 & 1.00 & 0.00 \\
\hline ATOM & 4 & $\mathrm{C}$ & unk & 1 & 0.738 & -3.085 & 3.320 & 1.00 & 0.00 \\
\hline ATOM & 5 & $\mathrm{C}$ & unk & 1 & 1.918 & -2.133 & 3.388 & 1.00 & 0.00 \\
\hline ATOM & 6 & $\mathrm{C}$ & unk & 1 & 2.712 & -1.952 & 2.311 & 1.00 & 0.00 \\
\hline ATOM & 7 & $\mathrm{C}$ & unk & 1 & 3.938 & -1.090 & 2.181 & 1.00 & 0.00 \\
\hline ATOM & 8 & $\mathrm{C}$ & unk & 1 & 3.727 & 0.150 & 1.268 & 1.00 & 0.00 \\
\hline ATOM & 9 & C & unk & 1 & 4.984 & 1.013 & 1.238 & 1.00 & 0.00 \\
\hline ATOM & 10 & $\mathrm{C}$ & unk & 1 & 5.847 & 1.160 & 0.213 & 1.00 & 0.00 \\
\hline ATOM & 11 & $\mathrm{C}$ & unk & 1 & 5.679 & 0.493 & -1.067 & 1.00 & 0.00 \\
\hline ATOM & 12 & $\mathrm{C}$ & unk & 1 & 5.010 & 0.950 & -2.147 & 1.00 & 0.00 \\
\hline ATOM & 13 & $\mathrm{C}$ & unk & 1 & 4.393 & 2.273 & -2.198 & 1.00 & 0.00 \\
\hline ATOM & 14 & $\mathrm{C}$ & unk & 1 & 3.555 & 2.719 & -3.149 & 1.00 & 0.00 \\
\hline ATOM & 15 & $\mathrm{C}$ & unk & 1 & 7.088 & 2.004 & 0.329 & 1.00 & 0.00 \\
\hline ATOM & 16 & $\mathrm{C}$ & unk & 1 & 2.547 & 1.013 & 1.726 & 1.00 & 0.00 \\
\hline ATOM & 17 & 0 & unk & 1 & 4.930 & -1.950 & 1.606 & 1.00 & 0.00 \\
\hline ATOM & 18 & $\mathrm{C}$ & unk & 1 & 2.112 & -1.462 & 4.722 & 1.00 & 0.00 \\
\hline ATOM & 19 & $\mathrm{C}$ & unk & 1 & -1.678 & -3.157 & 5.375 & 1.00 & 0.00 \\
\hline ATOM & 20 & 0 & unk & 1 & -2.451 & -0.388 & 3.213 & 1.00 & 0.00 \\
\hline ATOM & 21 & $\mathrm{C}$ & unk & 1 & -3.530 & 0.380 & 2.637 & 1.00 & 0.00 \\
\hline ATOM & 22 & $\mathrm{C}$ & unk & 1 & -3.047 & 1.856 & 2.500 & 1.00 & 0.00 \\
\hline ATOM & 23 & $\mathrm{C}$ & unk & 1 & -1.874 & 1.982 & 1.545 & 1.00 & 0.00 \\
\hline ATOM & 24 & $\mathrm{C}$ & unk & 1 & -1.858 & 2.776 & 0.464 & 1.00 & 0.00 \\
\hline ATOM & 25 & $\mathrm{C}$ & unk & 1 & -0.756 & 2.758 & -0.469 & 1.00 & 0.00 \\
\hline ATOM & 26 & $\mathrm{C}$ & unk & 1 & -0.557 & 3.568 & -1.530 & 1.00 & 0.00 \\
\hline ATOM & 27 & $\mathrm{C}$ & unk & 1 & -3.958 & -0.194 & 1.301 & 1.00 & 0.00 \\
\hline ATOM & 28 & $\mathrm{~N}$ & unk & 1 & -3.150 & -1.143 & 0.684 & 1.00 & 0.00 \\
\hline ATOM & 29 & $\mathrm{C}$ & unk & 1 & -3.646 & -1.485 & -0.488 & 1.00 & 0.00 \\
\hline ATOM & 30 & $\mathrm{~S}$ & unk & 1 & -5.124 & -0.707 & -0.905 & 1.00 & 0.00 \\
\hline ATOM & 31 & $\mathrm{C}$ & unk & 1 & -5.093 & 0.137 & 0.585 & 1.00 & 0.00 \\
\hline ATOM & 32 & $\mathrm{C}$ & unk & 1 & -3.024 & -2.490 & -1.431 & 1.00 & 0.00 \\
\hline ATOM & 33 & 0 & unk & 1 & -1.613 & -2.552 & -1.140 & 1.00 & 0.00 \\
\hline ATOM & 34 & $\mathrm{C}$ & unk & 1 & -0.846 & -1.634 & -1.766 & 1.00 & 0.00 \\
\hline ATOM & 35 & $\mathrm{~N}$ & unk & 1 & 0.438 & -1.774 & -1.337 & 1.00 & 0.00 \\
\hline ATOM & 36 & $\mathrm{C}$ & unk & 1 & 1.513 & -0.955 & -1.819 & 1.00 & 0.00 \\
\hline ATOM & 37 & $\mathrm{C}$ & unk & 1 & -3.613 & -3.890 & -1.183 & 1.00 & 0.00 \\
\hline ATOM & 38 & $\mathrm{C}$ & unk & 1 & -2.964 & -5.013 & -2.019 & 1.00 & 0.00 \\
\hline ATOM & 39 & $\mathrm{C}$ & unk & 1 & -3.127 & -4.790 & -3.522 & 1.00 & 0.00 \\
\hline ATOM & 40 & $\mathrm{C}$ & unk & 1 & -3.570 & -6.364 & -1.628 & 1.00 & 0.00 \\
\hline ATOM & 41 & $\mathrm{C}$ & unk & 1 & -2.649 & 2.433 & 3.862 & 1.00 & 0.00 \\
\hline ATOM & 42 & $\mathrm{C}$ & unk & 1 & -1.448 & 4.717 & -1.926 & 1.00 & 0.00 \\
\hline ATOM & 43 & 0 & unk & 1 & 2.630 & 4.357 & -4.568 & 1.00 & 0.00 \\
\hline ATOM & 44 & $\mathrm{C}$ & unk & 1 & 4.938 & 0.088 & -3.384 & 1.00 & 0.00 \\
\hline ATOM & 45 & 0 & unk & 1 & -3.937 & -1.869 & 4.147 & 1.00 & 0.00 \\
\hline ATOM & 46 & 0 & unk & 1 & -1.231 & -0.823 & -2.593 & 1.00 & 0.00 \\
\hline ATOM & 47 & $\mathrm{H}$ & unk & 1 & -0.661 & -1.737 & 2.331 & 1.00 & 0.00 \\
\hline ATOM & 48 & $\mathrm{H}$ & unk & 1 & 0.780 & -3.770 & 4.172 & 1.00 & 0.00 \\
\hline ATOM & 49 & $\mathrm{H}$ & unk & 1 & 0.811 & -3.724 & 2.431 & 1.00 & 0.00 \\
\hline ATOM & 50 & $\mathrm{H}$ & unk & 1 & 2.473 & -2.498 & 1.399 & 1.00 & 0.00 \\
\hline ATOM & 51 & $\mathrm{H}$ & unk & 1 & 4.321 & -0.782 & 3.158 & 1.00 & 0.00 \\
\hline ATOM & 52 & $\mathrm{H}$ & unk & 1 & 3.508 & -0.205 & 0.255 & 1.00 & 0.00 \\
\hline ATOM & 53 & $\mathrm{H}$ & unk & 1 & 5.197 & 1.540 & 2.168 & 1.00 & 0.00 \\
\hline ATOM & 54 & $\mathrm{H}$ & unk & 1 & 6.164 & -0.482 & -1.117 & 1.00 & 0.00 \\
\hline ATOM & 55 & $\mathrm{H}$ & unk & 1 & 4.654 & 2.953 & -1.389 & 1.00 & 0.00 \\
\hline ATOM & 56 & $\mathrm{H}$ & unk & 1 & 3.262 & 2.070 & -3.971 & 1.00 & 0.00 \\
\hline ATOM & 57 & $\mathrm{H}$ & unk & 1 & 7.983 & 1.395 & 0.162 & 1.00 & 0.00 \\
\hline ATOM & 58 & $\mathrm{H}$ & unk & 1 & 7.078 & 2.814 & -0.408 & 1.00 & 0.00 \\
\hline
\end{tabular}




\begin{tabular}{|c|c|c|c|c|c|c|c|c|c|}
\hline ATOM & 59 & $\mathrm{H}$ & unk & 1 & 7.180 & 2.461 & 1.320 & 1.00 & 0.00 \\
\hline ATOM & 60 & $\mathrm{H}$ & unk & 1 & 2.466 & 1.920 & 1.120 & 1.00 & 0.00 \\
\hline ATOM & 61 & $\mathrm{H}$ & unk & 1 & 1.599 & 0.475 & 1.626 & 1.00 & 0.00 \\
\hline ATOM & 62 & $\mathrm{H}$ & unk & 1 & 2.656 & 1.318 & 2.773 & 1.00 & 0.00 \\
\hline ATOM & 63 & $\mathrm{H}$ & unk & 1 & 5.735 & -1.415 & 1.502 & 1.00 & 0.00 \\
\hline ATOM & 64 & $\mathrm{H}$ & unk & 1 & 2.957 & -0.771 & 4.744 & 1.00 & 0.00 \\
\hline ATOM & 65 & $\mathrm{H}$ & unk & 1 & 1.223 & -0.884 & 4.993 & 1.00 & 0.00 \\
\hline ATOM & 66 & $\mathrm{H}$ & unk & 1 & 2.283 & -2.215 & 5.499 & 1.00 & 0.00 \\
\hline ATOM & 67 & $\mathrm{H}$ & unk & 1 & -2.408 & -3.966 & 5.267 & 1.00 & 0.00 \\
\hline ATOM & 68 & $\mathrm{H}$ & unk & 1 & -0.724 & -3.602 & 5.667 & 1.00 & 0.00 \\
\hline ATOM & 69 & $\mathrm{H}$ & unk & 1 & -1.994 & -2.517 & 6.206 & 1.00 & 0.00 \\
\hline ATOM & 70 & $\mathrm{H}$ & unk & 1 & -4.391 & 0.371 & 3.320 & 1.00 & 0.00 \\
\hline ATOM & 71 & $\mathrm{H}$ & unk & 1 & -3.885 & 2.454 & 2.118 & 1.00 & 0.00 \\
\hline ATOM & 72 & $\mathrm{H}$ & unk & 1 & -1.026 & 1.330 & 1.752 & 1.00 & 0.00 \\
\hline ATOM & 73 & $\mathrm{H}$ & unk & 1 & -2.711 & 3.414 & 0.253 & 1.00 & 0.00 \\
\hline ATOM & 74 & $\mathrm{H}$ & unk & 1 & -0.027 & 1.968 & -0.286 & 1.00 & 0.00 \\
\hline ATOM & 75 & $\mathrm{H}$ & unk & 1 & -5.876 & 0.835 & 0.848 & 1.00 & 0.00 \\
\hline ATOM & 76 & $\mathrm{H}$ & unk & 1 & -3.194 & -2.196 & -2.473 & 1.00 & 0.00 \\
\hline ATOM & 77 & $\mathrm{H}$ & unk & 1 & 0.593 & -2.464 & -0.616 & 1.00 & 0.00 \\
\hline ATOM & 78 & $\mathrm{H}$ & unk & 1 & 1.397 & -0.766 & -2.889 & 1.00 & 0.00 \\
\hline ATOM & 79 & $\mathrm{H}$ & unk & 1 & 1.499 & -0.006 & -1.278 & 1.00 & 0.00 \\
\hline ATOM & 80 & $\mathrm{H}$ & unk & 1 & 2.456 & -1.474 & -1.633 & 1.00 & 0.00 \\
\hline ATOM & 81 & $\mathrm{H}$ & unk & 1 & -3.484 & -4.145 & -0.121 & 1.00 & 0.00 \\
\hline ATOM & 82 & $\mathrm{H}$ & unk & 1 & -4.695 & -3.874 & -1.369 & 1.00 & 0.00 \\
\hline ATOM & 83 & $\mathrm{H}$ & unk & 1 & -1.891 & -5.055 & -1.793 & 1.00 & 0.00 \\
\hline ATOM & 84 & $\mathrm{H}$ & unk & 1 & -2.729 & -5.640 & -4.087 & 1.00 & 0.00 \\
\hline ATOM & 85 & $\mathrm{H}$ & unk & 1 & -4.182 & -4.669 & -3.793 & 1.00 & 0.00 \\
\hline ATOM & 86 & $\mathrm{H}$ & unk & 1 & -2.585 & -3.900 & -3.854 & 1.00 & 0.00 \\
\hline ATOM & 87 & $\mathrm{H}$ & unk & 1 & -3.093 & -7.180 & -2.182 & 1.00 & 0.00 \\
\hline ATOM & 88 & $\mathrm{H}$ & unk & 1 & -3.427 & -6.561 & -0.560 & 1.00 & 0.00 \\
\hline ATOM & 89 & $\mathrm{H}$ & unk & 1 & -4.644 & -6.394 & -1.838 & 1.00 & 0.00 \\
\hline ATOM & 90 & $\mathrm{H}$ & unk & 1 & -3.479 & 2.353 & 4.572 & 1.00 & 0.00 \\
\hline ATOM & 91 & $\mathrm{H}$ & unk & 1 & -1.785 & 1.915 & 4.292 & 1.00 & 0.00 \\
\hline ATOM & 92 & $\mathrm{H}$ & unk & 1 & -2.389 & 3.494 & 3.773 & 1.00 & 0.00 \\
\hline ATOM & 93 & $\mathrm{H}$ & unk & 1 & -0.868 & 5.641 & -2.010 & 1.00 & 0.00 \\
\hline ATOM & 94 & $\mathrm{H}$ & unk & 1 & -1.922 & 4.518 & -2.893 & 1.00 & 0.00 \\
\hline ATOM & 95 & $\mathrm{H}$ & unk & 1 & -2.245 & 4.915 & -1.207 & 1.00 & 0.00 \\
\hline ATOM & 96 & $\mathrm{H}$ & unk & 1 & 1.709 & 4.040 & -4.678 & 1.00 & 0.00 \\
\hline ATOM & 97 & $\mathrm{H}$ & unk & 1 & 5.365 & 0.611 & -4.247 & 1.00 & 0.00 \\
\hline ATOM & 98 & $\mathrm{H}$ & unk & 1 & 5.497 & -0.848 & -3.269 & 1.00 & 0.00 \\
\hline ATOM & 99 & $\mathrm{H}$ & unk & 1 & 3.902 & -0.184 & -3.611 & 1.00 & 0.00 \\
\hline ATOM & 100 & $\mathrm{C}$ & unk & 1 & 0.656 & 3.318 & -2.424 & 1.00 & 0.00 \\
\hline ATOM & 101 & $\mathrm{C}$ & unk & 1 & 1.764 & 4.398 & -2.283 & 1.00 & 0.00 \\
\hline ATOM & 102 & $\mathrm{C}$ & unk & 1 & 2.980 & 4.111 & -3.203 & 1.00 & 0.00 \\
\hline ATOM & 103 & $\mathrm{H}$ & unk & 1 & 3.778 & 4.829 & -2.979 & 1.00 & 0.00 \\
\hline ATOM & 104 & 0 & unk & 1 & 0.245 & 3.283 & -3.804 & 1.00 & 0.00 \\
\hline ATOM & 105 & $\mathrm{C}$ & unk & 1 & 2.212 & 4.584 & -0.829 & 1.00 & 0.00 \\
\hline ATOM & 106 & $\mathrm{H}$ & unk & 1 & 3.053 & 5.283 & -0.766 & 1.00 & 0.00 \\
\hline ATOM & 107 & $\mathrm{H}$ & unk & 1 & 1.406 & 4.999 & -0.216 & 1.00 & 0.00 \\
\hline ATOM & 108 & $\mathrm{H}$ & unk & 1 & 2.523 & 3.638 & -0.380 & 1.00 & 0.00 \\
\hline ATOM & 109 & $\mathrm{H}$ & unk & 1 & 1.347 & 5.355 & -2.627 & 1.00 & 0.00 \\
\hline ATOM & 110 & $\mathrm{H}$ & unk & 1 & 1.099 & 2.345 & -2.177 & 1.00 & 0.00 \\
\hline $\mathrm{ATOM}$ & 111 & $\mathrm{C}$ & unk & 1 & -0.392 & 2.055 & -4.155 & 1.00 & 0.00 \\
\hline ATOM & 112 & $\mathrm{H}$ & unk & 1 & -0.664 & 2.106 & -5.213 & 1.00 & 0.00 \\
\hline ATOM & 113 & $\mathrm{H}$ & unk & 1 & 0.296 & 1.217 & -4.017 & 1.00 & 0.00 \\
\hline ATOM & 114 & $\mathrm{H}$ & unk & 1 & -1.305 & 1.902 & -3.573 & 1.00 & 0.00 \\
\hline
\end{tabular}


PDB output-file for the conformer 5A of archzolid A

\begin{tabular}{|c|c|c|c|c|c|c|c|c|c|}
\hline HEADER & \multicolumn{9}{|c|}{ PROTEIN } \\
\hline COMPND & \multicolumn{9}{|c|}{ SMALL MOLECULE } \\
\hline AUTHOR & \multicolumn{9}{|c|}{ GENERATED BY MOLEKEL } \\
\hline ATOM & 1 & $\mathrm{C}$ & unk & 1 & -1.882 & -2.428 & 0.721 & 1.00 & 0.00 \\
\hline ATOM & 2 & $\mathrm{C}$ & unk & 1 & -0.688 & -2.330 & -0.174 & 1.00 & 0.00 \\
\hline $\mathrm{ATOM}$ & 3 & $\mathrm{C}$ & unk & 1 & 0.203 & -3.339 & -0.101 & 1.00 & 0.00 \\
\hline ATOM & 4 & $\mathrm{C}$ & unk & 1 & 1.491 & -3.474 & -0.863 & 1.00 & 0.00 \\
\hline ATOM & 5 & $\mathrm{C}$ & unk & 1 & 2.686 & -3.124 & -0.001 & 1.00 & 0.00 \\
\hline $\mathrm{ATOM}$ & 6 & $\mathrm{C}$ & unk & 1 & 3.365 & -1.975 & -0.207 & 1.00 & 0.00 \\
\hline ATOM & 7 & $\mathrm{C}$ & unk & 1 & 4.554 & -1.453 & 0.553 & 1.00 & 0.00 \\
\hline ATOM & 8 & $\mathrm{C}$ & unk & 1 & 4.388 & 0.035 & 0.972 & 1.00 & 0.00 \\
\hline $\mathrm{ATOM}$ & 9 & $\mathrm{C}$ & unk & 1 & 5.566 & 0.515 & 1.814 & 1.00 & 0.00 \\
\hline ATOM & 10 & $\mathrm{C}$ & unk & 1 & 6.622 & 1.260 & 1.428 & 1.00 & 0.00 \\
\hline ATOM & 11 & $\mathrm{C}$ & unk & 1 & 6.863 & 1.706 & 0.064 & 1.00 & 0.00 \\
\hline $\mathrm{ATOM}$ & 12 & $\mathrm{C}$ & unk & 1 & 6.124 & 2.550 & -0.684 & 1.00 & 0.00 \\
\hline ATOM & 13 & $\mathrm{C}$ & unk & 1 & 4.956 & 3.250 & -0.166 & 1.00 & 0.00 \\
\hline ATOM & 14 & C & unk & 1 & 3.905 & 3.637 & -0.904 & 1.00 & 0.00 \\
\hline $\mathrm{ATOM}$ & 15 & $\mathrm{C}$ & unk & 1 & 7.717 & 1.628 & 2.395 & 1.00 & 0.00 \\
\hline ATOM & 16 & $\mathrm{C}$ & unk & 1 & 3.099 & 0.273 & 1.772 & 1.00 & 0.00 \\
\hline ATOM & 17 & O & unk & 1 & 5.656 & -1.595 & -0.346 & 1.00 & 0.00 \\
\hline $\mathrm{ATOM}$ & 18 & $\mathrm{C}$ & unk & 1 & 3.050 & -4.158 & 1.032 & 1.00 & 0.00 \\
\hline ATOM & 19 & $\mathrm{C}$ & unk & 1 & -0.553 & -1.103 & -1.029 & 1.00 & 0.00 \\
\hline ATOM & 20 & 0 & unk & 1 & -2.766 & -1.460 & 0.403 & 1.00 & 0.00 \\
\hline $\mathrm{ATOM}$ & 21 & $\mathrm{C}$ & unk & 1 & -3.903 & -1.302 & 1.278 & 1.00 & 0.00 \\
\hline ATOM & 22 & C & unk & 1 & -3.576 & -0.344 & 2.477 & 1.00 & 0.00 \\
\hline ATOM & 23 & C & unk & 1 & -2.334 & 0.509 & 2.282 & 1.00 & 0.00 \\
\hline $\mathrm{ATOM}$ & 24 & $\mathrm{C}$ & unk & 1 & -2.345 & 1.675 & 1.617 & 1.00 & 0.00 \\
\hline ATOM & 25 & C & unk & 1 & -1.154 & 2.457 & 1.382 & 1.00 & 0.00 \\
\hline ATOM & 26 & $\mathrm{C}$ & unk & 1 & -1.028 & 3.479 & 0.510 & 1.00 & 0.00 \\
\hline $\mathrm{ATOM}$ & 27 & $\mathrm{C}$ & unk & 1 & -5.073 & -0.800 & 0.450 & 1.00 & 0.00 \\
\hline ATOM & 28 & $\mathrm{~N}$ & unk & 1 & -5.140 & 0.543 & 0.100 & 1.00 & 0.00 \\
\hline ATOM & 29 & C & unk & 1 & -6.201 & 0.788 & -0.641 & 1.00 & 0.00 \\
\hline $\mathrm{ATOM}$ & 30 & S & unk & 1 & -7.193 & -0.586 & -0.950 & 1.00 & 0.00 \\
\hline ATOM & 31 & $\mathrm{C}$ & unk & 1 & -6.110 & -1.565 & -0.054 & 1.00 & 0.00 \\
\hline ATOM & 32 & C & unk & 1 & -6.547 & 2.163 & -1.168 & 1.00 & 0.00 \\
\hline $\mathrm{ATOM}$ & 33 & 0 & unk & 1 & -6.881 & 2.011 & -2.563 & 1.00 & 0.00 \\
\hline ATOM & 34 & $\mathrm{C}$ & unk & 1 & -6.667 & 3.104 & -3.329 & 1.00 & 0.00 \\
\hline ATOM & 35 & $\mathrm{~N}$ & unk & 1 & -7.000 & 2.812 & -4.615 & 1.00 & 0.00 \\
\hline ATOM & 36 & $\mathrm{C}$ & unk & 1 & -6.867 & 3.763 & -5.685 & 1.00 & 0.00 \\
\hline ATOM & 37 & C & unk & 1 & -7.728 & 2.798 & -0.414 & 1.00 & 0.00 \\
\hline ATOM & 38 & $\mathrm{C}$ & unk & 1 & -7.500 & 3.033 & 1.093 & 1.00 & 0.00 \\
\hline ATOM & 39 & $\mathrm{C}$ & unk & 1 & -8.778 & 3.590 & 1.727 & 1.00 & 0.00 \\
\hline ATOM & 40 & C & unk & 1 & -6.334 & 3.985 & 1.364 & 1.00 & 0.00 \\
\hline ATOM & 41 & $\mathrm{C}$ & unk & 1 & -3.439 & -1.136 & 3.786 & 1.00 & 0.00 \\
\hline ATOM & 42 & $\mathrm{C}$ & unk & 1 & -2.115 & 3.972 & -0.414 & 1.00 & 0.00 \\
\hline ATOM & 43 & O & unk & 1 & 2.422 & 5.421 & -1.257 & 1.00 & 0.00 \\
\hline ATOM & 44 & $\mathrm{C}$ & unk & 1 & 6.529 & 2.830 & -2.111 & 1.00 & 0.00 \\
\hline ATOM & 45 & 0 & unk & 1 & -2.022 & -3.254 & 1.610 & 1.00 & 0.00 \\
\hline ATOM & 46 & 0 & unk & 1 & -6.245 & 4.179 & -2.933 & 1.00 & 0.00 \\
\hline ATOM & 47 & $\mathrm{H}$ & unk & 1 & 0.004 & -4.168 & 0.580 & 1.00 & 0.00 \\
\hline ATOM & 48 & $\mathrm{H}$ & unk & 1 & 1.585 & -4.509 & -1.218 & 1.00 & 0.00 \\
\hline ATOM & 49 & $\mathrm{H}$ & unk & 1 & 1.477 & -2.871 & -1.778 & 1.00 & 0.00 \\
\hline ATOM & 50 & $\mathrm{H}$ & unk & 1 & 3.033 & -1.307 & -1.006 & 1.00 & 0.00 \\
\hline ATOM & 51 & $\mathrm{H}$ & unk & 1 & 4.773 & -2.054 & 1.441 & 1.00 & 0.00 \\
\hline ATOM & 52 & $\mathrm{H}$ & unk & 1 & 4.319 & 0.627 & 0.056 & 1.00 & 0.00 \\
\hline $\mathrm{ATOM}$ & 53 & $\mathrm{H}$ & unk & 1 & 5.527 & 0.197 & 2.857 & 1.00 & 0.00 \\
\hline ATOM & 54 & $\mathrm{H}$ & unk & 1 & 7.761 & 1.273 & -0.378 & 1.00 & 0.00 \\
\hline ATOM & 55 & $\mathrm{H}$ & unk & 1 & 4.933 & 3.420 & 0.910 & 1.00 & 0.00 \\
\hline ATOM & 56 & $\mathrm{H}$ & unk & 1 & 3.864 & 3.415 & -1.965 & 1.00 & 0.00 \\
\hline ATOM & 57 & $\mathrm{H}$ & unk & 1 & 8.676 & 1.208 & 2.071 & 1.00 & 0.00 \\
\hline АТОM & 58 & $\mathrm{H}$ & unk & 1 & 7.817 & 2.716 & 2.463 & 1.00 & 0.00 \\
\hline
\end{tabular}




\begin{tabular}{|c|c|c|c|c|c|c|c|c|c|}
\hline ATOM & 59 & $\mathrm{H}$ & unk & 1 & 7.517 & 1.253 & 3.404 & 1.00 & 0.00 \\
\hline ATOM & 60 & $\mathrm{H}$ & unk & 1 & 3.046 & 1.307 & 2.133 & 1.00 & 0.00 \\
\hline ATOM & 61 & $\mathrm{H}$ & unk & 1 & 2.211 & 0.100 & 1.156 & 1.00 & 0.00 \\
\hline ATOM & 62 & $\mathrm{H}$ & unk & 1 & 3.040 & -0.390 & 2.642 & 1.00 & 0.00 \\
\hline ATOM & 63 & $\mathrm{H}$ & unk & 1 & 6.409 & -1.134 & 0.061 & 1.00 & 0.00 \\
\hline ATOM & 64 & $\mathrm{H}$ & unk & 1 & 4.037 & -4.003 & 1.472 & 1.00 & 0.00 \\
\hline ATOM & 65 & $\mathrm{H}$ & unk & 1 & 2.316 & -4.162 & 1.844 & 1.00 & 0.00 \\
\hline ATOM & 66 & $\mathrm{H}$ & unk & 1 & 3.068 & -5.155 & 0.578 & 1.00 & 0.00 \\
\hline ATOM & 67 & $\mathrm{H}$ & unk & 1 & 0.408 & -1.040 & -1.544 & 1.00 & 0.00 \\
\hline ATOM & 68 & $\mathrm{H}$ & unk & 1 & -1.337 & -1.081 & -1.792 & 1.00 & 0.00 \\
\hline ATOM & 69 & $\mathrm{H}$ & unk & 1 & -0.634 & -0.200 & -0.416 & 1.00 & 0.00 \\
\hline ATOM & 70 & $\mathrm{H}$ & unk & 1 & -4.206 & -2.283 & 1.669 & 1.00 & 0.00 \\
\hline ATOM & 71 & $\mathrm{H}$ & unk & 1 & -4.429 & 0.329 & 2.650 & 1.00 & 0.00 \\
\hline ATOM & 72 & $\mathrm{H}$ & unk & 1 & -1.393 & 0.112 & 2.660 & 1.00 & 0.00 \\
\hline ATOM & 73 & $\mathrm{H}$ & unk & 1 & -3.287 & 2.034 & 1.214 & 1.00 & 0.00 \\
\hline ATOM & 74 & $\mathrm{H}$ & unk & 1 & -0.285 & 2.184 & 1.980 & 1.00 & 0.00 \\
\hline ATOM & 75 & $\mathrm{H}$ & unk & 1 & -6.284 & -2.628 & 0.046 & 1.00 & 0.00 \\
\hline ATOM & 76 & $\mathrm{H}$ & unk & 1 & -5.647 & 2.783 & -1.077 & 1.00 & 0.00 \\
\hline ATOM & 77 & $\mathrm{H}$ & unk & 1 & -7.305 & 1.867 & -4.800 & 1.00 & 0.00 \\
\hline ATOM & 78 & $\mathrm{H}$ & unk & 1 & -7.544 & 3.477 & -6.492 & 1.00 & 0.00 \\
\hline ATOM & 79 & $\mathrm{H}$ & unk & 1 & -7.109 & 4.770 & -5.333 & 1.00 & 0.00 \\
\hline ATOM & 80 & $\mathrm{H}$ & unk & 1 & -5.835 & 3.742 & -6.044 & 1.00 & 0.00 \\
\hline ATOM & 81 & $\mathrm{H}$ & unk & 1 & -7.983 & 3.754 & -0.891 & 1.00 & 0.00 \\
\hline ATOM & 82 & $\mathrm{H}$ & unk & 1 & -8.621 & 2.173 & -0.552 & 1.00 & 0.00 \\
\hline ATOM & 83 & $\mathrm{H}$ & unk & 1 & -7.283 & 2.076 & 1.581 & 1.00 & 0.00 \\
\hline ATOM & 84 & $\mathrm{H}$ & unk & 1 & -8.649 & 3.727 & 2.806 & 1.00 & 0.00 \\
\hline ATOM & 85 & $\mathrm{H}$ & unk & 1 & -9.050 & 4.557 & 1.292 & 1.00 & 0.00 \\
\hline ATOM & 86 & $\mathrm{H}$ & unk & 1 & -9.617 & 2.902 & 1.578 & 1.00 & 0.00 \\
\hline ATOM & 87 & $\mathrm{H}$ & unk & 1 & -6.243 & 4.191 & 2.436 & 1.00 & 0.00 \\
\hline ATOM & 88 & $\mathrm{H}$ & unk & 1 & -5.383 & 3.554 & 1.038 & 1.00 & 0.00 \\
\hline ATOM & 89 & $\mathrm{H}$ & unk & 1 & -6.473 & 4.940 & 0.846 & 1.00 & 0.00 \\
\hline ATOM & 90 & $\mathrm{H}$ & unk & 1 & -4.349 & -1.712 & 3.986 & 1.00 & 0.00 \\
\hline ATOM & 91 & $\mathrm{H}$ & unk & 1 & -2.595 & -1.833 & 3.755 & 1.00 & 0.00 \\
\hline ATOM & 92 & $\mathrm{H}$ & unk & 1 & -3.283 & -0.462 & 4.635 & 1.00 & 0.00 \\
\hline ATOM & 93 & $\mathrm{H}$ & unk & 1 & -3.001 & 3.332 & -0.434 & 1.00 & 0.00 \\
\hline ATOM & 94 & $\mathrm{H}$ & unk & 1 & -2.432 & 4.976 & -0.116 & 1.00 & 0.00 \\
\hline ATOM & 95 & $\mathrm{H}$ & unk & 1 & -1.741 & 4.019 & -1.442 & 1.00 & 0.00 \\
\hline ATOM & 96 & $\mathrm{H}$ & unk & 1 & 3.224 & 5.972 & -1.278 & 1.00 & 0.00 \\
\hline ATOM & 97 & $\mathrm{H}$ & unk & 1 & 6.598 & 3.909 & -2.290 & 1.00 & 0.00 \\
\hline ATOM & 98 & $\mathrm{H}$ & unk & 1 & 7.508 & 2.401 & -2.352 & 1.00 & 0.00 \\
\hline ATOM & 99 & $\mathrm{H}$ & unk & 1 & 5.805 & 2.401 & -2.812 & 1.00 & 0.00 \\
\hline ATOM & 100 & $\mathrm{C}$ & unk & 1 & 0.284 & 4.251 & 0.382 & 1.00 & 0.00 \\
\hline ATOM & 101 & $\mathrm{C}$ & unk & 1 & 1.457 & 3.427 & -0.225 & 1.00 & 0.00 \\
\hline ATOM & 102 & $\mathrm{C}$ & unk & 1 & 2.699 & 4.343 & -0.361 & 1.00 & 0.00 \\
\hline ATOM & 103 & $\mathrm{H}$ & unk & 1 & 2.939 & 4.789 & 0.612 & 1.00 & 0.00 \\
\hline ATOM & 104 & 0 & unk & 1 & 0.697 & 4.679 & 1.696 & 1.00 & 0.00 \\
\hline ATOM & 105 & $\mathrm{C}$ & unk & 1 & 1.081 & 2.736 & -1.546 & 1.00 & 0.00 \\
\hline ATOM & 106 & $\mathrm{H}$ & unk & 1 & 0.170 & 2.140 & -1.447 & 1.00 & 0.00 \\
\hline ATOM & 107 & $\mathrm{H}$ & unk & 1 & 0.925 & 3.462 & -2.350 & 1.00 & 0.00 \\
\hline ATOM & 108 & $\mathrm{H}$ & unk & 1 & 1.866 & 2.031 & -1.852 & 1.00 & 0.00 \\
\hline ATOM & 109 & $\mathrm{H}$ & unk & 1 & 1.736 & 2.638 & 0.482 & 1.00 & 0.00 \\
\hline ATOM & 110 & $\mathrm{H}$ & unk & 1 & 0.122 & 5.141 & -0.241 & 1.00 & 0.00 \\
\hline ATOM & 111 & $\mathrm{C}$ & unk & 1 & 0.015 & 5.848 & 2.134 & 1.00 & 0.00 \\
\hline ATOM & 112 & $\mathrm{H}$ & unk & 1 & 0.389 & 6.106 & 3.128 & 1.00 & 0.00 \\
\hline ATOM & 113 & $\mathrm{H}$ & unk & 1 & 0.221 & 6.685 & 1.460 & 1.00 & 0.00 \\
\hline ATOM & 114 & $\mathrm{H}$ & unk & 1 & -1.062 & 5.675 & 2.205 & 1.00 & 0.00 \\
\hline
\end{tabular}


PDB output-file for the conformer 5B of archzolid A

\begin{tabular}{|c|c|c|c|c|c|c|c|c|c|}
\hline HEADER & & TF & & & & & & & \\
\hline COMPND & & & MOLEC & & & & & & \\
\hline AUTHOR & & $\mathrm{JER}$ & TED B & MOLEKEL & & & & & \\
\hline ATOM & 1 & $\mathrm{C}$ & UNK & 1 & -1.941 & -1.903 & 1.276 & 1.00 & 0.00 \\
\hline ATOM & 2 & $\mathrm{C}$ & UNK & 1 & -1.215 & -2.746 & 0.284 & 1.00 & 0.00 \\
\hline ATOM & 3 & $\mathrm{C}$ & UNK & 1 & -0.106 & -2.267 & -0.315 & 1.00 & 0.00 \\
\hline ATOM & 4 & $\mathrm{C}$ & UNK & 1 & 0.810 & -2.997 & -1.259 & 1.00 & 0.00 \\
\hline ATOM & 5 & $\mathrm{C}$ & UNK & 1 & 2.071 & -3.448 & -0.547 & 1.00 & 0.00 \\
\hline ATOM & 6 & $\mathrm{C}$ & UNK & 1 & 3.253 & -2.838 & -0.780 & 1.00 & 0.00 \\
\hline ATOM & 7 & $\mathrm{C}$ & UNK & 1 & 4.574 & -3.094 & -0.103 & 1.00 & 0.00 \\
\hline ATOM & 8 & $\mathrm{C}$ & UNK & 1 & 5.216 & -1.793 & 0.454 & 1.00 & 0.00 \\
\hline ATOM & 9 & $\mathrm{C}$ & UNK & 1 & 6.457 & -2.093 & 1.287 & 1.00 & 0.00 \\
\hline ATOM & 10 & $\mathrm{C}$ & UNK & 1 & 7.757 & -1.946 & 0.961 & 1.00 & 0.00 \\
\hline ATOM & 11 & $\mathrm{C}$ & UNK & 1 & 8.257 & -1.525 & -0.339 & 1.00 & 0.00 \\
\hline ATOM & 12 & $\mathrm{C}$ & UNK & 1 & 8.058 & -0.363 & -0.994 & 1.00 & 0.00 \\
\hline ATOM & 13 & $\mathrm{C}$ & UNK & 1 & 7.302 & 0.750 & -0.433 & 1.00 & 0.00 \\
\hline ATOM & 14 & $\mathrm{C}$ & UNK & 1 & 6.645 & 1.666 & -1.162 & 1.00 & 0.00 \\
\hline ATOM & 15 & $\mathrm{C}$ & UNK & 1 & 8.846 & -2.315 & 1.937 & 1.00 & 0.00 \\
\hline ATOM & 16 & $\mathrm{C}$ & UNK & 1 & 4.247 & -0.989 & 1.334 & 1.00 & 0.00 \\
\hline ATOM & 17 & 0 & UNK & 1 & 5.410 & -3.665 & -1.111 & 1.00 & 0.00 \\
\hline ATOM & 18 & $\mathrm{C}$ & UNK & 1 & 1.889 & -4.612 & 0.393 & 1.00 & 0.00 \\
\hline ATOM & 19 & $\mathrm{C}$ & UNK & 1 & -1.787 & -4.123 & 0.133 & 1.00 & 0.00 \\
\hline ATOM & 20 & 0 & UNK & 1 & -1.963 & -0.603 & 0.896 & 1.00 & 0.00 \\
\hline ATOM & 21 & $\mathrm{C}$ & UNK & 1 & -2.734 & 0.264 & 1.762 & 1.00 & 0.00 \\
\hline ATOM & 22 & $\mathrm{C}$ & UNK & 1 & -2.369 & 1.740 & 1.429 & 1.00 & 0.00 \\
\hline ATOM & 23 & $\mathrm{C}$ & UNK & 1 & -0.871 & 1.992 & 1.517 & 1.00 & 0.00 \\
\hline ATOM & 24 & $\mathrm{C}$ & UNK & 1 & -0.174 & 2.557 & 0.518 & 1.00 & 0.00 \\
\hline ATOM & 25 & $\mathrm{C}$ & UNK & 1 & 1.251 & 2.788 & 0.573 & 1.00 & 0.00 \\
\hline ATOM & 26 & $\mathrm{C}$ & UNK & 1 & 1.970 & 3.567 & -0.264 & 1.00 & 0.00 \\
\hline ATOM & 27 & $\mathrm{C}$ & UNK & 1 & -4.216 & 0.013 & 1.580 & 1.00 & 0.00 \\
\hline ATOM & 28 & $\mathrm{~N}$ & UNK & 1 & -4.757 & 0.059 & 0.301 & 1.00 & 0.00 \\
\hline ATOM & 29 & $\mathrm{C}$ & UNK & 1 & -6.042 & -0.232 & 0.334 & 1.00 & 0.00 \\
\hline ATOM & 30 & $S$ & UNK & 1 & -6.679 & -0.540 & 1.903 & 1.00 & 0.00 \\
\hline ATOM & 31 & $\mathrm{C}$ & UNK & 1 & -5.126 & -0.293 & 2.575 & 1.00 & 0.00 \\
\hline ATOM & 32 & $\mathrm{C}$ & UNK & 1 & -6.929 & -0.339 & -0.882 & 1.00 & 0.00 \\
\hline ATOM & 33 & 0 & UNK & 1 & -6.930 & 0.955 & -1.527 & 1.00 & 0.00 \\
\hline ATOM & 34 & $\mathrm{C}$ & UNK & 1 & -7.840 & 1.838 & -1.060 & 1.00 & 0.00 \\
\hline ATOM & 35 & $\mathrm{~N}$ & UNK & 1 & -7.681 & 3.021 & -1.713 & 1.00 & 0.00 \\
\hline ATOM & 36 & $\mathrm{C}$ & UNK & 1 & -8.480 & 4.179 & -1.427 & 1.00 & 0.00 \\
\hline ATOM & 37 & $\mathrm{C}$ & UNK & 1 & -6.356 & -1.336 & -1.906 & 1.00 & 0.00 \\
\hline ATOM & 38 & $\mathrm{C}$ & UNK & 1 & -6.258 & -2.798 & -1.427 & 1.00 & 0.00 \\
\hline ATOM & 39 & $\mathrm{C}$ & UNK & 1 & -5.561 & -3.646 & -2.495 & 1.00 & 0.00 \\
\hline ATOM & 40 & $\mathrm{C}$ & UNK & 1 & -7.628 & -3.397 & -1.106 & 1.00 & 0.00 \\
\hline ATOM & 41 & $\mathrm{C}$ & UNK & 1 & -3.079 & 2.726 & 2.366 & 1.00 & 0.00 \\
\hline ATOM & 42 & $\mathrm{C}$ & UNK & 1 & 1.386 & 4.426 & -1.359 & 1.00 & 0.00 \\
\hline ATOM & 43 & 0 & UNK & 1 & 6.150 & 3.955 & $-1 \cdot 321$ & 1.00 & 0.00 \\
\hline ATOM & 44 & $\mathrm{C}$ & UNK & 1 & 8.663 & -0.177 & -2.365 & 1.00 & 0.00 \\
\hline ATOM & 45 & 0 & UNK & 1 & -2.465 & -2.368 & 2.278 & 1.00 & 0.00 \\
\hline ATOM & 46 & 0 & UNK & 1 & -8.670 & 1.613 & -0.194 & 1.00 & 0.00 \\
\hline ATOM & 47 & $\mathrm{H}$ & UNK & 1 & 0.223 & -1.254 & -0.089 & 1.00 & 0.00 \\
\hline ATOM & 48 & $\mathrm{H}$ & UNK & 1 & 0.327 & -3.857 & -1.733 & 1.00 & 0.00 \\
\hline ATOM & 49 & $\mathrm{H}$ & UNK & 1 & 1.051 & -2.315 & -2.085 & 1.00 & 0.00 \\
\hline ATOM & 50 & $\mathrm{H}$ & UNK & 1 & 3.288 & -2.029 & -1.512 & 1.00 & 0.00 \\
\hline ATOM & 51 & $\mathrm{H}$ & UNK & 1 & 4.487 & -3.827 & 0.704 & 1.00 & 0.00 \\
\hline ATOM & 52 & $\mathrm{H}$ & UNK & 1 & 5.481 & -1.170 & -0.404 & 1.00 & 0.00 \\
\hline ATOM & 53 & $\mathrm{H}$ & UNK & 1 & 6.232 & -2.475 & 2.285 & 1.00 & 0.00 \\
\hline ATOM & 54 & $\mathrm{H}$ & UNK & 1 & 8.884 & -2.281 & -0.815 & 1.00 & 0.00 \\
\hline ATOM & 55 & $\mathrm{H}$ & UNK & 1 & 7.220 & 0.785 & 0.652 & 1.00 & 0.00 \\
\hline ATOM & 56 & $\mathrm{H}$ & UNK & 1 & 6.651 & 1.611 & -2.245 & 1.00 & 0.00 \\
\hline ATOM & 57 & $\mathrm{H}$ & UNK & 1 & 9.465 & -3.126 & 1.539 & 1.00 & 0.00 \\
\hline ATOM & 58 & $\mathrm{H}$ & UNK & 1 & 9.490 & -1.451 & 2.134 & 1.00 & 0.00 \\
\hline
\end{tabular}




\begin{tabular}{|c|c|c|c|c|c|c|c|c|c|}
\hline ATOM & 59 & $\mathrm{H}$ & UNK & 1 & 8.443 & -2.650 & 2.898 & 1.00 & 0.00 \\
\hline ATOM & 60 & $\mathrm{H}$ & UNK & 1 & 4.744 & -0.114 & 1.768 & 1.00 & 0.00 \\
\hline ATOM & 61 & $\mathrm{H}$ & UNK & 1 & 3.394 & -0.618 & 0.756 & 1.00 & 0.00 \\
\hline ATOM & 62 & $\mathrm{H}$ & UNK & 1 & 3.856 & -1.597 & 2.157 & 1.00 & 0.00 \\
\hline ATOM & 63 & $\mathrm{H}$ & UNK & 1 & 6.317 & -3.656 & -0.760 & 1.00 & 0.00 \\
\hline ATOM & 64 & $\mathrm{H}$ & UNK & 1 & 2.830 & -5.095 & 0.668 & 1.00 & 0.00 \\
\hline ATOM & 65 & $\mathrm{H}$ & UNK & 1 & 1.392 & -4.288 & 1.313 & 1.00 & 0.00 \\
\hline ATOM & 66 & $\mathrm{H}$ & UNK & 1 & 1.275 & -5.387 & -0.078 & 1.00 & 0.00 \\
\hline ATOM & 67 & $\mathrm{H}$ & UNK & 1 & -1.291 & -4.713 & -0.641 & 1.00 & 0.00 \\
\hline ATOM & 68 & $\mathrm{H}$ & UNK & 1 & -1.704 & -4.679 & 1.073 & 1.00 & 0.00 \\
\hline ATOM & 69 & $\mathrm{H}$ & UNK & 1 & -2.847 & -4.063 & -0.140 & 1.00 & 0.00 \\
\hline ATOM & 70 & $\mathrm{H}$ & UNK & 1 & -2.443 & 0.066 & 2.803 & 1.00 & 0.00 \\
\hline ATOM & 71 & $\mathrm{H}$ & UNK & 1 & -2.697 & 1.953 & 0.402 & 1.00 & 0.00 \\
\hline ATOM & 72 & $\mathrm{H}$ & UNK & 1 & -0.375 & 1.714 & 2.445 & 1.00 & 0.00 \\
\hline ATOM & 73 & $\mathrm{H}$ & UNK & 1 & -0.700 & 2.841 & -0.389 & 1.00 & 0.00 \\
\hline ATOM & 74 & $\mathrm{H}$ & UNK & 1 & 1.782 & 2.260 & 1.366 & 1.00 & 0.00 \\
\hline ATOM & 75 & $\mathrm{H}$ & UNK & 1 & -4.951 & -0.395 & 3.636 & 1.00 & 0.00 \\
\hline ATOM & 76 & $\mathrm{H}$ & UNK & 1 & -7.954 & -0.633 & -0.629 & 1.00 & 0.00 \\
\hline ATOM & 77 & $\mathrm{H}$ & UNK & 1 & -6.919 & 3.067 & -2.374 & 1.00 & 0.00 \\
\hline ATOM & 78 & $\mathrm{H}$ & UNK & 1 & -9.499 & 3.887 & -1.157 & 1.00 & 0.00 \\
\hline ATOM & 79 & $\mathrm{H}$ & UNK & 1 & -8.023 & 4.720 & -0.594 & 1.00 & 0.00 \\
\hline ATOM & 80 & $\mathrm{H}$ & UNK & 1 & -8.498 & 4.817 & -2.314 & 1.00 & 0.00 \\
\hline ATOM & 81 & $\mathrm{H}$ & UNK & 1 & -6.971 & -1.290 & -2.816 & 1.00 & 0.00 \\
\hline ATOM & 82 & $\mathrm{H}$ & UNK & 1 & -5.361 & -0.993 & -2.222 & 1.00 & 0.00 \\
\hline ATOM & 83 & $\mathrm{H}$ & UNK & 1 & -5.641 & -2.844 & -0.521 & 1.00 & 0.00 \\
\hline ATOM & 84 & $\mathrm{H}$ & UNK & 1 & -5.452 & -4.683 & -2.159 & 1.00 & 0.00 \\
\hline ATOM & 85 & $\mathrm{H}$ & UNK & 1 & -6.128 & -3.651 & -3.432 & 1.00 & 0.00 \\
\hline ATOM & 86 & $\mathrm{H}$ & UNK & 1 & -4.558 & -3.259 & -2.705 & 1.00 & 0.00 \\
\hline ATOM & 87 & $\mathrm{H}$ & UNK & 1 & -7.535 & -4.454 & -0.832 & 1.00 & 0.00 \\
\hline ATOM & 88 & $\mathrm{H}$ & UNK & 1 & -8.101 & -2.886 & -0.263 & 1.00 & 0.00 \\
\hline ATOM & 89 & $\mathrm{H}$ & UNK & 1 & -8.302 & -3.330 & -1.967 & 1.00 & 0.00 \\
\hline ATOM & 90 & $\mathrm{H}$ & UNK & 1 & -2.772 & 3.757 & 2.157 & 1.00 & 0.00 \\
\hline ATOM & 91 & $\mathrm{H}$ & UNK & 1 & -4.166 & 2.685 & 2.240 & 1.00 & 0.00 \\
\hline ATOM & 92 & $\mathrm{H}$ & UNK & 1 & -2.850 & 2.515 & 3.416 & 1.00 & 0.00 \\
\hline ATOM & 93 & $\mathrm{H}$ & UNK & 1 & 0.295 & 4.406 & -1.403 & 1.00 & 0.00 \\
\hline ATOM & 94 & $\mathrm{H}$ & UNK & 1 & 1.672 & 5.472 & -1.202 & 1.00 & 0.00 \\
\hline ATOM & 95 & $\mathrm{H}$ & UNK & 1 & 1.759 & 4.115 & -2.339 & 1.00 & 0.00 \\
\hline ATOM & 96 & $\mathrm{H}$ & UNK & 1 & 7.107 & 4.079 & -1.193 & 1.00 & 0.00 \\
\hline ATOM & 97 & $\mathrm{H}$ & UNK & 1 & 9.236 & 0.756 & -2.414 & 1.00 & 0.00 \\
\hline ATOM & 98 & $\mathrm{H}$ & UNK & 1 & 9.353 & -0.988 & -2.625 & 1.00 & 0.00 \\
\hline ATOM & 99 & $\mathrm{H}$ & UNK & 1 & 7.885 & -0.156 & -3.135 & 1.00 & 0.00 \\
\hline ATOM & 100 & $\mathrm{C}$ & UNK & 1 & 3.490 & 3.696 & -0.128 & 1.00 & 0.00 \\
\hline ATOM & 101 & $\mathrm{C}$ & UNK & 1 & 4.280 & 2.498 & -0.727 & 1.00 & 0.00 \\
\hline ATOM & 102 & $\mathrm{C}$ & UNK & 1 & 5.801 & 2.772 & -0.601 & 1.00 & 0.00 \\
\hline ATOM & 103 & $\mathrm{H}$ & UNK & 1 & 6.063 & 2.943 & 0.450 & 1.00 & 0.00 \\
\hline ATOM & 104 & 0 & UNK & 1 & 3.835 & 3.779 & 1.270 & 1.00 & 0.00 \\
\hline ATOM & 105 & $\mathrm{C}$ & UNK & 1 & 3.855 & 2.165 & -2.166 & 1.00 & 0.00 \\
\hline ATOM & 106 & $\mathrm{H}$ & UNK & 1 & 2.782 & 1.963 & -2.232 & 1.00 & 0.00 \\
\hline ATOM & 107 & $\mathrm{H}$ & UNK & 1 & 4.096 & 2.980 & -2.856 & 1.00 & 0.00 \\
\hline ATOM & 108 & $\mathrm{H}$ & UNK & 1 & 4.343 & 1.249 & -2.519 & 1.00 & 0.00 \\
\hline ATOM & 109 & $\mathrm{H}$ & UNK & 1 & 4.080 & 1.606 & -0.122 & 1.00 & 0.00 \\
\hline ATOM & 110 & $\mathrm{H}$ & UNK & 1 & 3.818 & 4.620 & -0.623 & 1.00 & 0.00 \\
\hline ATOM & 111 & $\mathrm{C}$ & UNK & 1 & 3.700 & 5.097 & 1.789 & 1.00 & 0.00 \\
\hline ATOM & 112 & $\mathrm{H}$ & UNK & 1 & 3.958 & 5.073 & 2.852 & 1.00 & 0.00 \\
\hline ATOM & 113 & $\mathrm{H}$ & UNK & 1 & 4.391 & 5.778 & 1.282 & 1.00 & 0.00 \\
\hline ATOM & 114 & $\mathrm{H}$ & UNK & 1 & 2.673 & 5.458 & 1.690 & 1.00 & 0.00 \\
\hline
\end{tabular}

